\title{
Divergent acyl carrier protein decouples mitochondrial Fe-S cluster biogenesis from fatty acid synthesis in malaria parasites
}

\author{
Seyi Falekun ${ }^{1}$, Jaime Sepulveda ${ }^{1}$, Yasaman Jami-Alahmadi ${ }^{2}$, Hahnbeom Park ${ }^{3}$, \\ James A Wohlschlegel ${ }^{2}$, Paul A Sigala1*
}

${ }^{1}$ Department of Biochemistry, University of Utah School of Medicine, Salt Lake City, United States; ${ }^{2}$ Department of Biological Chemistry, University of California, Los Angeles, Los Angeles, United States; ${ }^{3}$ Department of Biochemistry, University of Washington, Seattle, United States

*For correspondence: p.sigala@biochem.utah.edu

Competing interest: The authors declare that no competing interests exist.

Funding: See page 21

Preprinted: 13 April 2021

Received: 25 June 2021

Accepted: 06 October 2021

Published: 06 October 2021

Reviewing Editor: Malcolm J McConville, The University of Melbourne, Australia

(c) Copyright Falekun et al. This article is distributed under the terms of the Creative Commons Attribution License, which permits unrestricted use and redistribution provided that the original author and source are credited.

\begin{abstract}
Most eukaryotic cells retain a mitochondrial fatty acid synthesis (FASII) pathway whose acyl carrier protein (mACP) and 4-phosphopantetheine (Ppant) prosthetic group provide a soluble scaffold for acyl chain synthesis and biochemically couple FASIl activity to mitochondrial electron transport chain (ETC) assembly and Fe-S cluster biogenesis. In contrast, the mitochondrion of Plasmodium falciparum malaria parasites lacks FASIl enzymes yet curiously retains a divergent $\mathrm{mACP}$ lacking a Ppant group. We report that ligand-dependent knockdown of mACP is lethal to parasites, indicating an essential FASII-independent function. Decyl-ubiquinone rescues parasites temporarily from death, suggesting a dominant dysfunction of the mitochondrial ETC. Biochemical studies reveal that Plasmodium mACP binds and stabilizes the Isd11-Nfs1 complex required for Fe-S cluster biosynthesis, despite lacking the Ppant group required for this association in other eukaryotes, and knockdown of parasite mACP causes loss of Nfs1 and the Rieske Fe-S protein in ETC complex III. This work reveals that Plasmodium parasites have evolved to decouple mitochondrial Fe-S cluster biogenesis from FASIl activity, and this adaptation is a shared metabolic feature of other apicomplexan pathogens, including Toxoplasma and Babesia. This discovery unveils an evolutionary driving force to retain interaction of mitochondrial Fe-S cluster biogenesis with ACP independent of its eponymous function in FASII.
\end{abstract}

\section{Introduction}

Malaria is an ancient scourge of humanity and remains a pressing global health challenge, especially in tropical Africa where hundreds of thousands of people die from malaria each year. Plasmodium falciparum malaria parasites are single-celled eukaryotes that evolved under unique selective pressures with unusual metabolic adaptations compared to human cells and well-studied model organisms such as yeast. Understanding the unique biochemical pathways that specialize parasites for growth within human red blood cells will shed light on their evolutionary divergence from other eukaryotes and unveil new parasite-specific targets for development of novel antimalarial therapies.

$P$. falciparum retains an essential mitochondrion required for biosynthesis of pyrimidines, acetyl-CoA, and Fe-S clusters (Painter et al., 2007; Jhun et al., 2018; van Dooren et al., 2006; Oppenheim et al., 2014; Gisselberg et al., 2013). Although the parasite mitochondrion also contains enzymes involved in the citric acid cycle and biosynthesis of ATP and heme, these pathways are dispensable for blood-stage parasites, which can scavenge host heme and obtain sufficient ATP from cytoplasmic 
glycolysis (Ke et al., 2014; Nagaraj et al., 2013; Ke et al., 2015; Sturm et al., 2015; MacRae et al., 2013). In addition to these pathways, most eukaryotes, including mammals and yeast, also contain a mitochondrial type II fatty acid biosynthesis (FASII) pathway that generates the octanoate precursor of the lipoic acid cofactor used by several mitochondrial dehydrogenases (Hiltunen et al., 2009). In contrast to human and yeast cells, FASIl enzymes in P. falciparum have been lost by the mitochondrion and are retained instead by the apicoplast organelle (Figure 1-figure supplement 1; Shears et al., 2015). Although critical lipoate-dependent enzymes are present in the parasite mitochondrion (Jhun et al., 2018), prior work has shown that these enzymes utilize scavenged lipoate obtained from the red blood cell rather than de novo synthesis (Allary et al., 2007).

The acyl carrier protein (ACP) is a key component of FASII and contains a strictly conserved Ser residue modified by a 4-phosphopantetheine (Ppant) group whose terminal thiol tethers the nascent acyl chain during fatty acid initiation, modification, and elongation (Chan and Vogel, 2010). Consistent with FASII targeting to the apicoplast in P. falciparum, this organelle features a well-studied ACP homolog (PF3D7_0208500, aACP) that retains canonical ACP features, including the conserved Ser modified by a 4-Ppant group required for FASII function (Shears et al., 2015; Gallagher and Prigge, 2010). Although apicoplast FASII activity is essential for P. falciparum growth within mosquitoes and the human liver, this pathway is dispensable for blood-stage parasites, which can scavenge fatty acids from the host (Shears et al., 2015; van Schaijk et al., 2014; Yu et al., 2008).

Despite loss of mitochondrial FASII enzymes, $P$. falciparum has curiously retained a second ACP homolog (PF3D7_1208300) that is annotated as a mitochondrial ACP (mACP) but has not been directly studied. We noted that recent genome-wide knock-out studies in Plasmodium berghei and $P$. falciparum both reported that this gene was refractory to disruption, suggesting an essential, FASIIindependent function (Zhang et al., 2018; Gomes et al., 2015). We became interested to unravel what this essential function might be. Recent studies in yeast and human cells have identified an expanding network of mitochondrial ACP interactions beyond FASII that includes roles in respiratory chain assembly, Fe-S cluster biogenesis, and mitochondrial ribosomal translation (Van Vranken et al., 2016; Van Vranken et al., 2018; Nowinski et al., 2020; Brown et al., 2017; Majmudar et al., 2019). However, in all cases these critical interactions in yeast and/or humans are linked to mitochondrial FASII function and the Ppant group of ACP (Brown et al., 2017; Cory et al., 2017; Angerer et al., 2014; Boniecki et al., 2017).

We localized mACP to the P. falciparum mitochondrion and set out to understand its FASIIindependent function in this organelle. Using conditional knockdown and immunoprecipitation (IP) studies, we discovered that $\mathrm{mACP}$ is essential for parasite viability and plays a critical role in binding and stabilizing the Isd11-Nfs1 cysteine desulfurase complex required for mitochondrial Fe-S cluster biogenesis. Unlike mACP interactions in yeast and humans, $P$. falciparum mACP binds to Isd11 via a divergent molecular interface that does not involve a 4-Ppant group. This work unveils a new molecular paradigm for essential mACP function without a Ppant group, underscores the critical and conserved role of $\mathrm{mACP}$ in mitochondrial Fe-S cluster biogenesis, and highlights a Plasmodium-specific adaptation suitable for exploration as a metabolic vulnerability for parasite-directed antimalarial therapy.

\section{Results}

\section{Mitochondrial ACP is essential for $P$. falciparum despite loss of FASII in this organelle}

The $P$. falciparum genome encodes two ACP homologs that include the well-studied protein targeted to the apicoplast (aACP, PF3D7_0208500) and a second homolog annotated as a mitochondrial ACP (mACP, PF3D7_1208300). Unlike the apicoplast ACP, which retains the conserved Ser for 4-Ppant attachment, $\mathrm{mACP}$ has curiously replaced this Ser with a Phe residue that cannot be modified by a 4-Ppant group (Figure 1A and Figure 1-figure supplement 2). This Ser-to-Phe substitution in mACP is consistent with the loss of mitochondrial FASII and the ACP-modifying phosphopantetheine transferase enzyme (Figure 1-figure supplement 1) and suggested a non-canonical function for mACP.

To test if mACP localized to the parasite mitochondrion, we created a Dd2 P. falciparum line that episomally expressed mACP fused to a $\mathrm{C}$-terminal dual hemagglutinin (HA) tag. Immunofluorescence analysis of this $m A C P-H A_{2}$ line revealed strong colocalization between $m A C P-H A_{2}$ and the mitochondrial marker, HSP60 (Figure 1B, Figure 1-figure supplement 3). On the basis of this colocalization, 


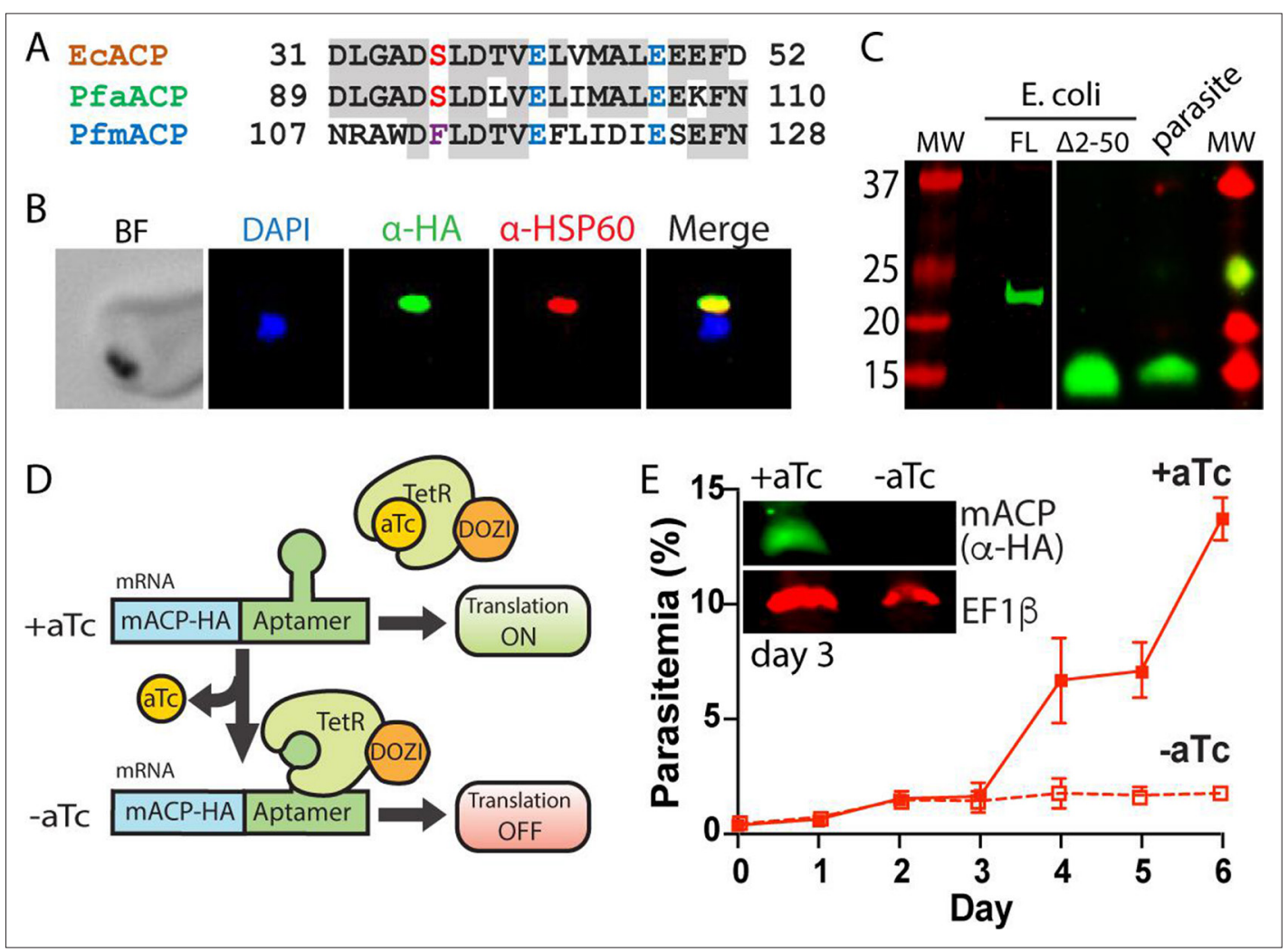

Figure 1. Mitochondrial ACP is essential for blood-stage P. falciparum. (A) Partial sequence alignment of ACP homologs from E. coli and the P. falciparum apicoplast (aACP) and mitochondrion (mACP). The conserved Ser residue that is modified by a 4-Ppant group is in red, the divergent Phe residue of $\mathrm{mACP}$ is in purple, and conserved Glu residues are in blue. (B) Immunofluorescence microscopy of fixed Dd2 parasites episomally expressing mACP-HA $\mathrm{A}_{2}$ and stained with DAPI (nucleus, blue), anti-HA (green), and anti-HSP60 (mitochondrion, red) antibodies ( $\mathrm{BF}=$ bright field). (C) Anti-HA western blot $(\mathrm{WB})$ analysis of cellular lysates of $E$. coli expressing full-length (FL) or truncated ( $\triangle 2-50) \mathrm{mACP}-\mathrm{HA}_{2}$ and Dd2 P. falciparum parasites episomally expressing fulllength $\mathrm{mACP}-\mathrm{HA}_{2}$. The left and right images are from different WB experiments ( $10 \%$ gels) that were aligned by molecular weight (MW) markers. (D) Schematic depiction of protein translational regulation using the aptamer/ TetR-DOZI system. Normal protein expression occurs in the presence but not absence of anhydrotetracycline (aTc). (E) Continuous growth assay of synchronous mACP-HA/FLAG-aptamer/TetR-DOZI parasites in the presence or absence of aTc. Data points and error bars are the average and standard deviation from two biological replicates. Inset is an anti-HA and anti-EF1 $\beta$ (37 kDa) WB of parasite lysates harvested on day 3 of the continuous growth assay. WBs were repeated $2-3$ times.

The online version of this article includes the following source data and figure supplement(s) for figure 1:

Figure supplement 1. P. falciparum homologs of E. coli FASII proteins.

Figure supplement 1-source data 1. Source file for Figure 1-figure supplement 1.

Figure supplement 2. Sequence and mass spectrometry (MS) analyses of ACP.

Figure supplement 3. Additional immunofluorescence microscopy images $m A C P-H A_{2}$

Figure supplement 4. Schematic depiction of $m A C P$ gene editing and verifying genomic integration by PCR.

Figure supplement 5. Giemsa-stained blood smears of mACP-aptamer/TetR-DOZI parasites cultured for $120 \mathrm{hr}$ (5 days) \pm anhydrotetracycline (aTc).

we conclude that $\mathrm{mACP}$ is indeed targeted to the parasite mitochondrion, as predicted. Full-length $\mathrm{mACP}-\mathrm{HA}_{2}$ has a predicted molecular mass $\sim 21 \mathrm{kDa}$, but SDS-polyacrylamide gel electrophoresis (SDS-PAGE) and western blot (WB) analysis of immunoprecipitated $\mathrm{mACP}_{-} \mathrm{HA}_{2}$ indicated that this protein migrated with an apparent molecular mass $\sim 15 \mathrm{kDa}$ (Figure 1C). This lower size suggested that $\mathrm{mACP}$ was likely to be post-translationally processed upon mitochondrial import, which would be consistent with known processing of mitochondrial ACP in yeast and humans (Vögtle et al., 2009; Vaca Jacome et al., 2015). We extended our WB analysis to include mACP-HA $A_{2}$ that was expressed 
heterologously in Escherichia coli, where proteolytic processing is not expected. The recombinant, fulllength $\mathrm{mACP}-\mathrm{HA}_{2}$ expressed in bacteria migrated with an apparent molecular mass close to $21 \mathrm{kDa}$, as expected. Based on sequence homology, the known ACP-processing sites in yeast and humans (Vögtle et al., 2009; Vaca Jacome et al., 2015), and mass spectrometry (MS) analysis of parasiteexpressed mACP (Figure 1-figure supplement 2), we identified Leu-51 as the possible N-terminus of mature $\mathrm{mACP}$ in $P$. falciparum. We therefore cloned and bacterially expressed a truncated $(\Delta 2-50)$ $m A C P-H A_{2}$ beginning with Leu-51 (after a start Met). This truncated mACP protein co-migrated by SDS-PAGE with $m A C P-H A_{2}$ expressed in parasites (Figure 1C), suggesting that Leu-51 is at or near the $\mathrm{N}$-terminus of mature $\mathrm{mACP}$ in parasites. We conclude that $\mathrm{mACP}$ is proteolytically processed upon import into the $P$. falciparum mitochondrion.

To directly test if $\mathrm{mACP}$ is essential for blood-stage parasite growth and viability, we used CRISPR/ Cas9 to tag the mACP gene in Dd2 parasites to encode a C-terminal HA-FLAG epitope tag and the aptamer/TetR-DOZI system that enables anhydrotetracycline (aTc)-dependent control of protein expression (Figure 1D; Ganesan et alo, 2016). Correct integration into the mACP locus was confirmed by PCR analysis of polyclonal and clonal parasite lines (Figure 1-figure supplement 4). To evaluate mACP knockdown and its impact on parasite growth, we synchronized parasites to the ring stage, split these parasites into two equal populations $\pm \mathrm{aTc}$, and monitored parasite growth over multiple $48 \mathrm{hr}$ growth cycles. Parasites grew indistinguishably \pm aTc for the first 3 days. However, parasites grown without aTc displayed a major growth defect on day 4 in the third intraerythrocytic lifecycle (Figure 1E), similar to prior studies with this knockdown system (Ganesan et al., 2016). Blood-smear analysis on day 5 indicated widespread parasite death (Figure 1-figure supplement 5). WB analysis of parasite samples harvested in the second cycle, after 3 days of growth -aTc, indicated robust knockdown of mACP expression relative to parasites grown $+\mathrm{aTc}$ (Figure 1E). We conclude that mACP is essential for $P$. falciparum parasites during blood-stage growth.

\section{Mitochondrial ACP binds the Isd11-Nfs1 complex required for Fe-S cluster biogenesis}

Recent studies in yeast and human cells have revealed that mitochondrial ACP binds to a variety of small, three-helical adapter proteins that bear a conserved Leu-Tyr-Arg (LYR) sequence motif on their $\mathrm{N}$-terminal helix (Majmudar et al., 2019; Angerer, 2015). The Leu mediates an intramolecular helicalhelical contact while the Tyr and Arg side chains interact with conserved acidic residues on ACP (Cory et al., 2017; Boniecki et al., 2017; Herrera et al., 2019). These LYR-motif proteins mediate diverse mitochondrial processes that include respiratory chain assembly, Fe-S cluster biogenesis, and ribosomal translation (Van Vranken et al., 2016; Van Vranken et al., 2018; Brown et al., 2017; Angerer et al., 2014). Given the strong conservation of mitochondrial LYR proteins and ACP interactions across eukaryotes, we reasoned that the essential function of $P$. falciparum $\mathrm{mACP}$ might involve interaction with a conserved LYR protein.

Using the sequences of known LYR proteins from yeast and humans as bait (Angerer, 2015), we conducted a BLAST search of the P. falciparum genome to look for possible homologs. In contrast to other eukaryotes, $P$. falciparum lacked identifiable homologs of LYR proteins that mediate respiratory chain assembly (LYRM3/NDUFB9, LYRM6/NDUFA6, LYRM7/MZM1, LYRM8/SDHAF1, ACN9/SDHAF3, and $\mathrm{FMC1/C7orf55)} \mathrm{or} \mathrm{interactions} \mathrm{with} \mathrm{mitochondrial} \mathrm{ribosomes} \mathrm{(LOR8F8).} \mathrm{However,} \mathrm{we} \mathrm{identified}$ a clear homolog for Isd11 (PF3D7_1311000, e-value 3e-6), which functions in Fe-S cluster biogenesis. Our BLAST analysis suggested that Isd11 is the only LYR-protein homolog retained by parasites (Figure 2-figure supplement 1).

P. falciparum Isd11 is $29 \%$ identical to human Isd11 (also known as LYRM4), including conservation of the LYR sequence motif near the $\mathrm{N}$-terminus (Figure 2A). The parasite mACP retains the conserved Glu residues ( 5 and 11 amino acids C-terminal to the modified Ser in canonical ACPs) known to interact with the Tyr and Arg residues of the LYR motif on Isd11 at the mACP-Isd11 binding interface (Figure 1A; Cory et al., 2017; Boniecki et al., 2017). In yeast and humans, Isd11 directly binds and stabilizes Nfs1, a mitochondrial cysteine desulfurase required for biogenesis of Fe-S clusters (Wiedemann et al., 2006). Mitochondrial ACP in these organisms directly binds Isd11 to stabilize the mACP-Isd11-Nfs1 complex (Figure 2B), with loss of ACP and/or Isd11 resulting in Nfs1 instability and defective Fe-S cluster biogenesis (Van Vranken et al., 2016; Lill and Freibert, 2020; Herrera et alo, 2018; Cai et al., 2017). P. falciparum retains a mitochondrial Nfs1 homolog (PF3D7_0727200) that 


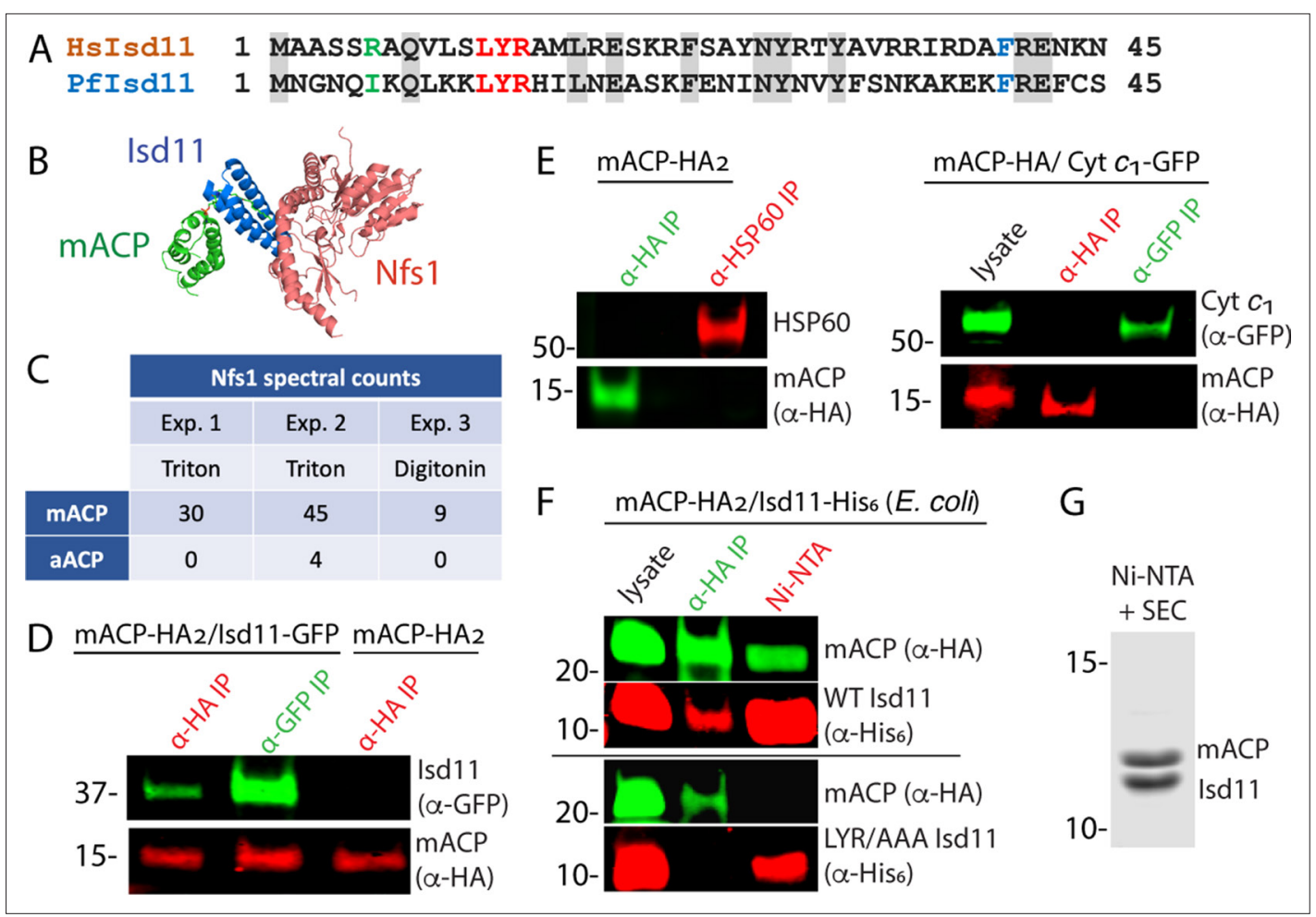

Figure 2. Mitochondrial ACP binds the Isd11-Nfs1 complex. (A) Partial sequence alignment of human and $P$. falciparum Isd11. The conserved LYR sequence motif is in red, the conserved Phe residue is in blue, and residue 6 is in green. (B) X-ray structural model of the mACP-Isd11-Nfs1 complex (PDB entry 5USR). For simplicity, only a single copy of each protein is shown, rather than the functional dimer (Boniecki et al., 2017). (C) Table of spectral counts for $P$. falciparum Nfs1 detected by tandem mass spectrometry for anti-HA immunoprecipitation (IP) studies of lysates from Dd2 parasites episomally expressing $\mathrm{mACP}_{-} \mathrm{HA}_{2}$ or $\mathrm{aACP}-\mathrm{HA}_{2}$. Parasites were lysed in either Triton $\mathrm{X}-100$ or digitonin. Similar spectral counts for $\mathrm{mACP}$ and AACP bait proteins were detected in each experiment (Figure 2-figure supplement 5). (D) Anti-HA or anti-GFP co-immunoprecipitation (co-IP)/western blot (WB) studies of Dd2 parasites episomally expressing mACP-HA $A_{2}$ with or without Isd11-GFP. (E) Anti-HA, anti-HSP60, or anti-GFP co-IP/WB studies of Dd2 parasites episomally expressing $m A C P-H A_{2}$ or endogenously expressing $m A C P$ HA (knockdown line) and episomally expressing cyt $c_{1}$-GFP. (F) Anti-HA co-IP or nickel-nitrilotriacetic acid (Ni-NTA) pulldown and WB studies of E. coli bacteria recombinantly expressing full-length mACP-HA ${ }_{2}$ and Isd11-His 6 (WT or LYR/AAA mutant) and probed with anti-HA and anti-His 6 antibodies. All lanes were loaded with equivalent sample volumes, are from the same blot, and were processed identically but were cropped from non-contiguous lanes. (G) Coomassie-stained SDS-PAGE gel of recombinant $\mathrm{His}_{6}$-Isd11 and $\triangle 2-50 \mathrm{mACP}-\mathrm{HA}_{2}$ purified from E. coli by

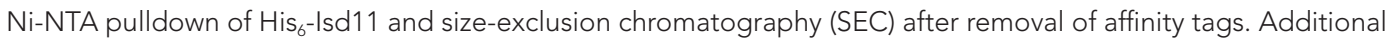
images for WB experiments are shown in Figure 2-figure supplement 3. WBs are representative of 2-3 replicate experiments with independent samples.

The online version of this article includes the following source data and figure supplement(s) for figure 2:

Figure supplement 1. BLAST analysis of LYR-motif protein homologs found in P. falciparum.

Figure supplement 1-source data 1. Source file for Figure 2-figure supplement 1.

Figure supplement 2. Specific enrichment of $\mathrm{Nfs} 1$ in anti-HA-tag IP of $m A C P-H A_{2}$ relative to anti-HA-tag IP aACP$\mathrm{HA}_{2}$.

Figure supplement 3. Co-localization of Isd11-GFP and MitoTracker in Dd2 parasites expressing mACP-HA $\mathrm{A}_{2}$ and Isd11-GFP.

Figure supplement 4. Additional images for western blot (WB) analyses.

Figure supplement 5. Spectral counts for $P$. falciparum mACP or aACP detected by tandem mass spectrometry.

Figure supplement 5-source data 1. Source file for Figure 2-figure supplement 5.

Figure supplement 6. Mass spectrometry (MS) analysis of recombinant parasite Isd11 and mACP expressed in bacteria.

Figure supplement 7. P. falciparum Isd11 does not stably bind to apicoplast ACP. 
is predicted from genome-wide knock-out studies to be essential (Gomes et al., 2015; Gisselberg et al., 2013) but whose role in Fe-S cluster biogenesis has not been studied. Collectively, conservation of Isd11 and Nfs1 in P. falciparum, the conserved role for ACP in stabilizing the mACP-Isd11-Nfs1 complex in other eukaryotes, and retention of the LYR sequence motif in $P$. falciparum Isd11 strongly suggested that mACP was likely to interact with Isd11 to form the mACP-Isd11-Nfs1 complex in parasites.

To identify protein interaction partners of $m A C P$, we used anti-HA IP to isolate $m A C P-H A_{2}$ from parasites followed by tandem MS to identify parasite proteins that co-purified with mACP. In multiple independent pulldowns, we identified $\mathrm{Nfs} 1$ as strongly enriched in the mACP-HA $\mathrm{A}_{2}$ sample compared

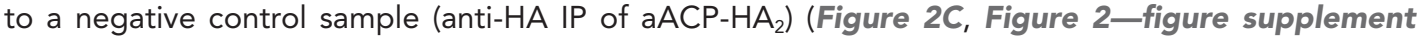
2). We did not observe peptides corresponding to Isd11 in mACP-HA $A_{2} I P / M S$ samples. However, its absence is not unexpected given the short Isd11 length (87 residues) that leads to comparatively few tryptic peptides.

To directly test for $\mathrm{mACP}$ interaction with Isd11, we transfected the $\mathrm{mACP}-\mathrm{HA}_{2} / \mathrm{Dd} 2$ line with an episome encoding expression of Isd11 with a C-terminal GFP tag that was previously used to localize Isd11 to the mitochondrion (Gisselberg et al., 2013). We confirmed that Isd11-GFP expressed in this line colocalized with MitoTracker (Figure 2-figure supplement 3). Using this Dd2 line expressing mACP-HA 2 and Isd11-GFP, we performed reciprocal IP/WB experiments that confirmed stable interaction of these two proteins in parasites (Figure 2D, Figure 2-figure supplement 4). This interaction appeared to be specific as mACP did not co-IP with the abundant mitochondrial chaperone, HSP60, or with GFP-tagged cytochrome $c_{1}$ (Figure 2E, Figure 2-figure supplement 4). Because these IP experiments could not distinguish whether mACP binding to Isd11 was direct or mediated by other parasite proteins, we turned to heterologous studies in E. coli. Reciprocal IP experiments with lysates from bacteria coexpressing P. falciparum mACP-HA $\mathrm{H}_{2}$ and Isd11-His ${ }_{6}$ confirmed stable interaction of these proteins in the absence of other parasite-specific factors (Figure 2F). In experiments with Isd11 LYR mutants, however, this interaction was strongly reduced (YR/AA) or eliminated (LYR/AAA), despite similar expression of all recombinant proteins (Figure 2F, Figure 2-figure supplement 4). Clarified lysates from bacteria coexpressing truncated $(\triangle 2-50) \mathrm{mACP}_{-} \mathrm{HA} \mathrm{A}_{2}$ and Isd11-His ${ }_{6}$ were also fractionated by passage over Ni-NTA resin to selectively pull down His-tagged Isd11 and interactors followed by size-exclusion chromatography. Analysis by Coomassie-stained SDS-PAGE and tandem MS indicated robust isolation of a 1:1 complex containing Isd11 and mACP (Figure 2G, Figure 2-figure supplement 6). We conclude that mACP directly binds to Isd11 and that this interaction involves the LYR motif.

Yeast and human Isd11 retain a conserved Arg-6 residues upstream of the LYR motif (Figure 2A) that electrostatically stabilizes the negatively charged oxygen atoms of the Ppant group in the ACPIsd11 complex (Cory et al., 2017; Boniecki et al., 2017; Herrera et al., 2019), and mutation of this Arg ablates binding of human Isd11 to ACP (Majmudar et al., 2019). Because parasite mACP lacks the negatively charged Ppant group and has replaced it with a hydrophobic Phe, retention of Arg-6 by $P$. falciparum Isd11 would be predicted to destabilize binding of these two proteins. The parasite Isd11, however, has replaced Arg-6 with an Ile residue (Figure 2A) expected to more favorably interact with the local hydrophobic surface of mACP created by Ser-to-Phe substitution. These sequence features suggest that parasite Isd11 has co-evolved with mACP to optimize formation of the mACP-Isd11 complex in P. falciparum in the absence of FASII and an acyl-Ppant group on mACP. Consistent with this model, recombinantly parasite Isd11 expressed in bacteria did not co-purify with the endogenous $\sim 9 \mathrm{kDa}$ E. coli ACP (Figure 2G) or with coexpressed apicoplast ACP (Figure 2figure supplement 7), both of which retain the conserved Ser and Ppant modification. This specificity by $P$. falciparum Isd11 contrasts with that of human Isd11, which robustly binds bacterial ACP when expressed in E. coli (Cory et al., 2017; Boniecki et al., 2017; Cai et al., 2017).

\section{Structural modeling of the mACP-Isd11 interface}

Association of Isd11 and mACP in yeast and human cells depends critically on the acyl-Ppant group of mACP (Figure 3A), with little or no association observed in these cells between Isd11 and the mACP Ser-to-Ala mutant that lacks a Ppant group (Van Vranken et al., 2016; Van Vranken et al., 2018; Majmudar et al., 2019). The mACP-Isd11 interface in these organisms also includes key electrostatic interactions between conserved Lys and Arg residues in Isd11 and Glu and Asp residues in 


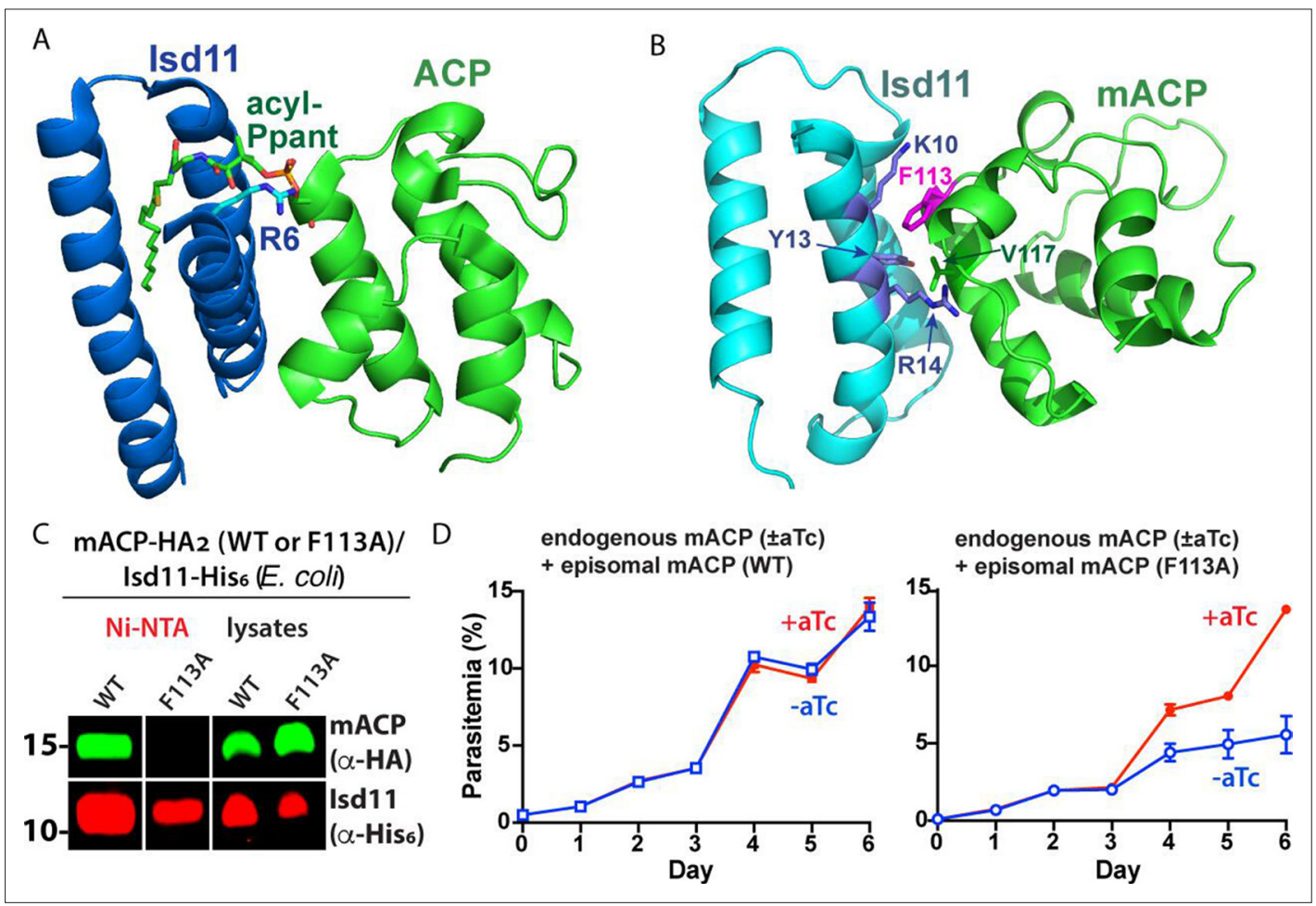

Figure 3. Structural modeling and functional tests of the divergent Phe113 residue of parasite mACP. (A) X-ray crystallographic structure of human Isd11 bound to E. coli ACP highlighting the central role of the acyl-Ppant group of ACP and R6 residue of Isd11 in stabilizing this complex (PDB entry 5USR). (B) Rosetta-based, energyminimized structural model of the P. falciparum mACP-Isd11 interface. Conserved electrostatic interactions that also contribute to the binding interface are shown in Figure 3-figure supplement 1. The Rosetta model is included as Figure 3-source data 1. (C) Nickel-nitrilotriacetic acid (Ni-NTA) pulldown and WB studies of E. coli bacteria recombinantly expressing $\triangle 2-50$ mACP-HA $A_{2}$ (WT or F113A mutant) and Isd11-His ${ }_{6}$ and probed with anti-HA and anti-His ${ }_{6}$ antibodies. All lanes were loaded with equivalent sample volumes, are from the same blot, and were processed identically but were cropped from non-contiguous lanes. The uncropped WB is shown in Figure 2-figure supplement 3. (D) Continuous growth assays of synchronous mACP-aptamer/ TetR-DOZI parasites episomally expressing WT or F113A mACP-HA $\mathrm{A}_{2}$ and grown in the presence or absence of anhydrotetracycline (aTc). Data points and error bars are the average and standard deviation from two biological replicates. The presence of an HA epitope tag on both the endogenous and episomal mACP prevented determination of endogenous mACP expression differences \pm aTc by WB. WBs are representative of 2-3 replicate experiments with independent samples.

The online version of this article includes the following figure supplement(s) for figure 3:

Source data 1. PDB file of energy-minimized Rosetta model of the $P$. falciparum Isd11-mACP complex.

Figure supplement 1. Conserved electrostatic interactions at the mACP-Isd11 interface.

Figure supplement 2. Uncropped image of western blot (WB) probing interaction of WT or F113A mACP-HA $A_{2}$ and Isd11-His 6 in E. coli.

mACP (Figure 3-figure supplement 1; Cory et al., 2017). As an initial step to understand the divergent molecular features of the $P$. falciparum mACP-Isd11 interface that stabilize complexation in the absence of an acyl-Ppant group, we generated a homology model of this complex using published structures and then carried out energy optimization and refinement using the Rosetta software (Park et al., 2018). The resulting low-energy structural model suggested that the unusual Phe113 residue of mACP partitions into a hydrophobic pocket at the mACP-Isd11 interface composed of the aliphatic side-chain methylene groups of Lys10 and Arg14 and the phenyl ring of Tyr13 of Isd11 as well as Val117 on mACP (Figure 3B). The predicted binding interface also included conserved electrostatic interactions between basic residues on parasite Isd11 and acidic groups on mACP (Figure 3-figure supplement 1). 
To test if the divergent Phe113 residue of $\mathrm{mACP}$ contributes substantially to its association with Isd11, we heterologously coexpressed Isd11-His ${ }_{6}$ and $\Delta 2-50 \mathrm{mACP}-\mathrm{HA}_{2}$ (WT or the F113A mutant) in $E$. coli and observed robust expression of all recombinant proteins in bacterial lysates. We then used Ni-NTA to selectively pull down His-tagged Isd11 and observed that WT mACP-HA 2 but not the F113A mutant co-purified with Isd11 (Figure 3C, Figure 3-figure supplement 2). These results indicate that mutation of Phe113 to Ala substantially weakens the mACP-Isd11 interaction, supporting the conclusion that Phe113 contributes to the mACP-Isd11 binding interface in the mACP-Isd11-Nfs 1 complex as suggested by structural modeling (Figure 3B).

To test the functional impact of the Phe113Ala mACP mutation on parasite growth, we transfected our mACP knockdown parasite line with episomes encoding either WT or F113A mACP. Parasites expressing a second copy of WT mACP showed identical growth in the presence or absence of aTc. In contrast, parasites that episomally expressed the F113A mACP mutant showed strongly diminished growth upon aTc removal and loss of endogenous mACP (Figure 3D). These results support the conclusion that the Phe residue of $\mathrm{mACP}$ plays a key role in stabilizing the mACP-Isd11 association required for $\mathrm{Nfs} 1$ stability and function.

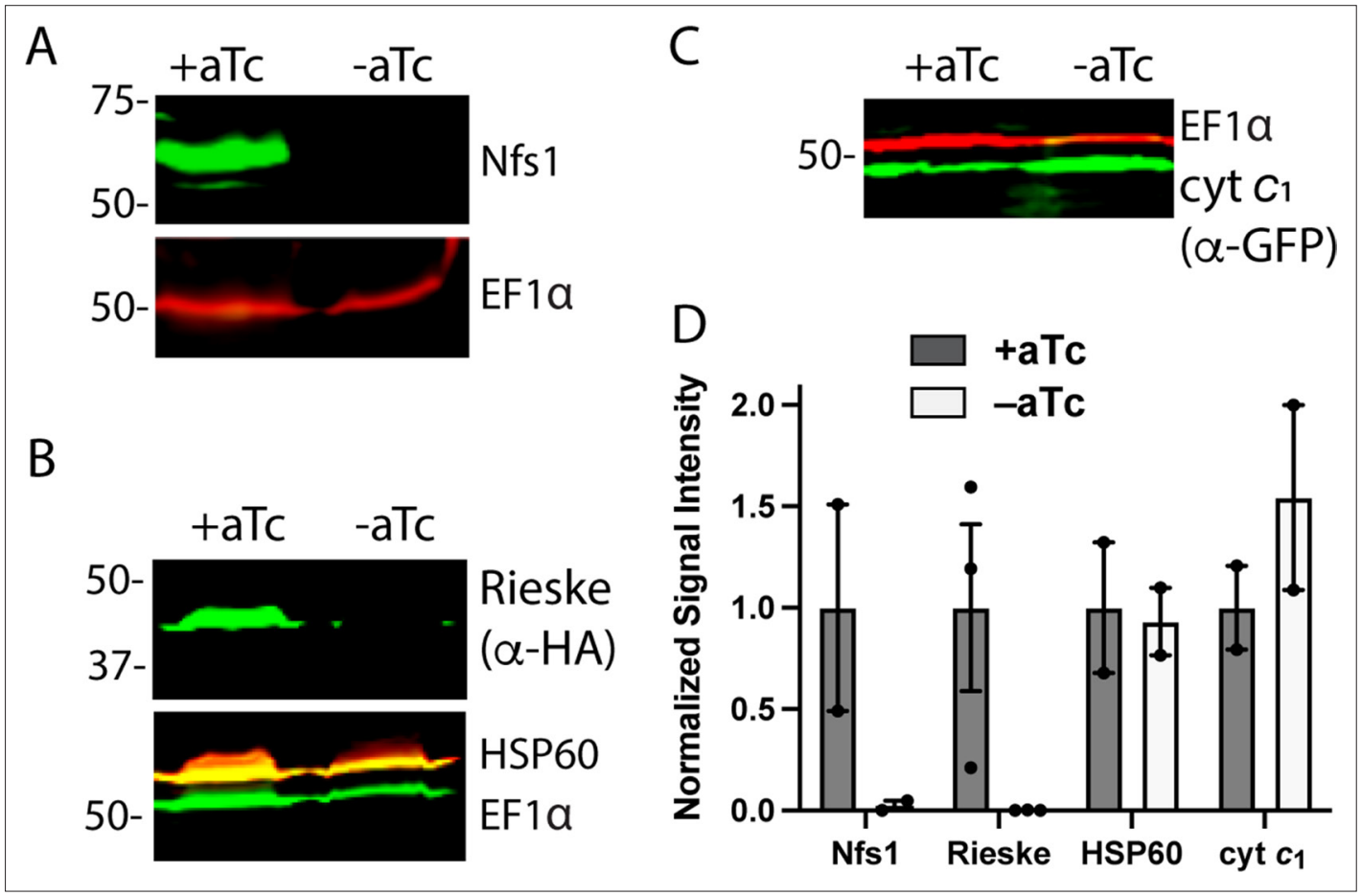

Figure 4. Loss of mACP specifically destabilizes Nfs1 and the Rieske Fe-S protein. Western blot analysis of equivalent volumes of lysates from $\mathrm{mACP}$-aptamer/TetR-DOZI Dd2 parasites without or with episomal expression of Rieske-HA $\mathrm{A}_{2}$ or cyt $c_{1}$-GFP that were grown \pm aTc for 3 days and probed for cytosolic EF1 $\alpha(\sim 50 \mathrm{kDa})$ and $(\mathbf{A}) \mathrm{Nfs} 1$, (B) HA-tag and HSP60, or or (C) GFP. For (B), the blot was first probed with rat anti-HA and rabbit anti-HSP60 $1^{\circ}$ antibodies, imaged after probing with anti-rat and anti-rabbit $2^{\circ}$ antibodies, probed additionally with rabbit anti-EF1 $\alpha$ antibody, and imaged again after probing with anti-rabbit $2^{\circ}$ antibody. The diminished intensity of EF1 $\alpha$ in the -aTc relative to + aTc samples likely reflects the slowing growth of these late second-cycle parasites cultured -aTc due to reduced fitness from loss of mACP. (D) Mean \pm SEM of the integrated signal intensity for each indicated protein under \pm aTc conditions relative to the loading control (EF1 $\alpha$ or EF1 $\beta$ ) and normalized to + aTc conditions. Black circles are individual data points from 2-3 replicate experiments with independent samples. Normalized signal intensity values for $+\mathrm{aTc}$ versus -aTc conditions were analyzed by two-tailed unpaired t-test to determine the following p values: Nfs1 (0.2), Rieske-HA $(0.07), \mathrm{HSP6O}(0.9)$, and cyt $c_{1}(0.4)$. Uncropped blots are shown in Figure 4-source data 1.

The online version of this article includes the following figure supplement(s) for figure 4:

Source data 1. Raw integrated signal intensity values for western blot densitometry analysis.

Figure supplement 1. Validation of anti-Nfs1 antibody for specific recognition of the parasite Nfs1 homolog. 


\section{Loss of mACP destabilizes Nfs1 and the Rieske protein in ETC complex III}

$\mathrm{Nfs} 1$ stability in other eukaryotes depends critically on its association with Isd11 and ACP, with loss of ACP resulting in degradation of Nfs1 and lethal defects in Fe-S cluster biogenesis (Van Vranken et alı, 2016; Cory et al., 2017; Boniecki et al., 2017; Lill and Freibert, 2020; Cai et al., 2017). Loss of Nfs1 upon mACP knockdown would therefore be sufficient to explain mACP essentiality in $P$. falciparum. To test the impact of mACP knockdown on Nfs1 protein levels in parasites, we used a commercial anti-Nfs1 antibody (Abcam 229829) raised against a 250-amino acid region of human Nfs1 that is $55 \%$ identical to $P$. falciparum Nfs 1 and that selectively recognized recombinant $P$. falciparum $\mathrm{Nfs} 1$ expressed in E. coli (Figure 4-figure supplement 1). We grew our mACP aptamer/TetR-DOZI parasites \pm aTc, harvested them at the end of the second intraerythrocytic growth cycle before parasite death, and analyzed expression of $\mathrm{Nfs} 1$ by WB using this $\mathrm{Nfs} 1$ antibody.

In parasites grown $+\mathrm{aTc}$, the anti-Nfs1 antibody recognized a band at $\sim 60 \mathrm{kDa}$ (Figure $4 \mathrm{~A}$ ) that is close to the predicted molecular mass of $63 \mathrm{kDa}$ for this protein. WB analysis of parasites grown in -aTc conditions failed to detect this $\sim 60 \mathrm{kDa}$ band (Figure $4 \mathrm{~A}$ ), strongly suggesting that $\mathrm{Nfs} 1$ stability is tightly coupled to mACP expression and that loss of mACP results in Nfs1 degradation in parasites as it does in yeast (Van Vranken et al., 2016). Loss of Nfs1 upon mACP knockdown suggested that parasite death was likely to involve dysfunction in one or more Fe-S cluster-dependent pathways. We set out to understand which pathway defect(s) might explain parasite death.

The mitochondrial iron-sulfur cluster (ISC) biogenesis pathway supplies Fe-S clusters to various mitochondrial proteins and has been suggested from studies in yeast to produce a key intermediate that is exported out of the organelle and elaborated by cytoplasmic proteins to assemble and deliver Fe-S clusters to client proteins in the cytoplasm and nucleus (Lill and Freibert, 2020; Kispal et al., 1999; Sharma et al., 2010; Rouault and Tong, 2005). Multiple Fe-S cluster proteins function within the $P$. falciparum mitochondrion (Haussig et al., 2014). Aconitase (PF3D7_1342100) and succinate dehydrogenase (PF3D7_1212800) are dispensable for blood-stage parasite growth (Ke et alı, 2015). Ferredoxin (PF3D7_1214600) is expected to be essential (Gomes et al., 2015), but its main role is to provide electrons for the ISC pathway (Lill and Freibert, 2020). Class I fumarate hydratase (PF3D7_0927300), which functions in the citric acid cycle but may also contribute to purine scavenging, was refractory to disruption in $P$. falciparum (Ke et al., 2015) but successfully deleted in $P$. berghei in a mouse strain-dependent manner (Jayaraman et al., 2018). The Rieske protein (PF3D7_1439400), which is an essential component of ETC complex III (Zhang et al., 1998), is the only known mitochondrial Fe-S cluster protein that has an unequivocally essential function apart from the ISC pathway. We therefore focused on understanding the effect of mACP knockdown on the Rieske protein as a prior study in yeast showed that mACP knockdown resulted in loss of Rieske (Van Vranken et al., 2016).

To probe the status of Rieske upon mACP knockdown in parasites, we transfected the mACPaptamer/TetR-DOZI parasites with an episome encoding Rieske-HA $\mathrm{A}_{2}$. A band corresponding to the epitope-tagged Rieske was detected $\sim 43 \mathrm{kDa}$ in WB analysis of parasites grown + aTc. This band, however, was undetectable in parasites grown $72 \mathrm{hr}$ in -aTc conditions to downregulate mACP expression (Figure 4B). These results indicate that knockdown of $\mathrm{mACP}$ and subsequent degradation of $\mathrm{Nfs} 1$ are accompanied by loss of the Fe-S cluster-dependent Rieske protein. The impact of mACP knockdown on stability of Nfs1 and Rieske was specific to these proteins as loss of mACP in -aTc conditions did not reduce expression levels of the mitochondrial chaperone HSP60 or the integral ETC complex III component, cyt $c_{1}$ (Figure $4 B$ and C).

\section{Loss of mACP causes ETC failure and sensitizes parasites to mitochondrial depolarization by proguanil}

Since the Rieske protein is an essential electron-transfer component of ETC complex III (Zhang et al., 1998), we reasoned that loss of $\mathrm{mACP}$ and Rieske likely results in ETC failure. In blood-stage parasites, which rely on glycolysis rather than oxidative phosphorylation for ATP synthesis, the essential function of the mitochondrial ETC is to oxidatively recycle the ubiquinone cofactor used by several dehydrogenases, of which dihydroorotate dehydrogenase (DHOD) is most critical (Painter et alo, 2007; Ke et al., 2011). Prior work has shown that exogenous addition of the soluble ubiquinone analog, decylubiquinone (dQ), rescues parasites from ETC dysfunction caused by the ETC complex III inhibitor, 


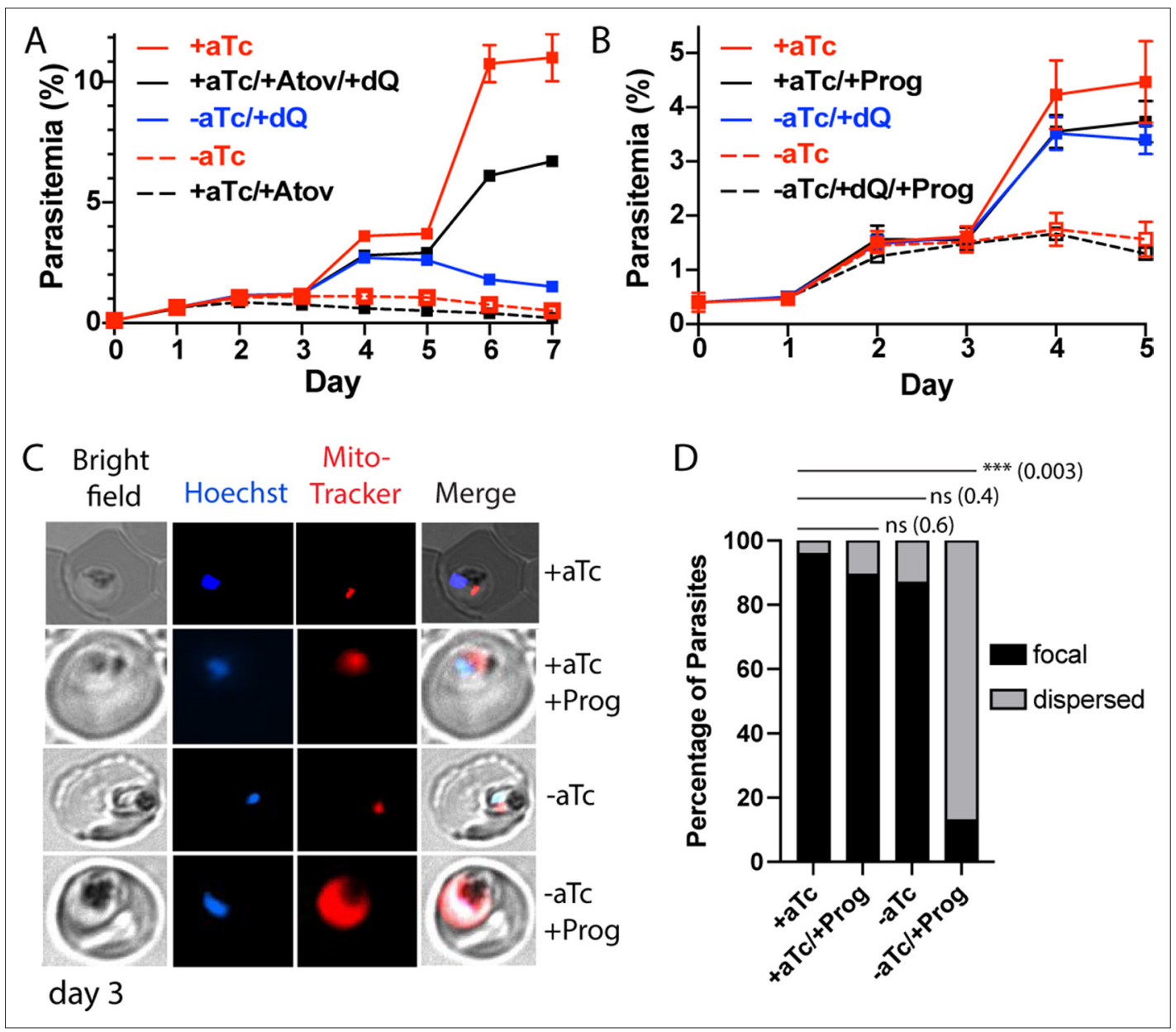

Figure 5. Loss of $m A C P$ causes electron transport chain (ETC) failure and sensitizes parasites to proguanil. Continuous growth assays of synchronous mACP-aptamer/TetR-DOZI parasites grown $(\mathbf{A}) \pm$ anhydrotetracycline (aTc), \pm atovaquone (Atov, $100 \mathrm{nM}), \pm$ decyl-ubiquinone $(\mathrm{dQ}, 15 \mu \mathrm{M})$, and (B) \pm proguanil (Prog, $1 \mu \mathrm{M})$. Data points and error bars are the average and standard deviation from 2 to 3 biological replicates. (C) Fluorescence microscopy images of live mACP-aptamer/TetR-DOZI Dd2 parasites cultured 3 days \pm aTc, 2 days $\pm 5 \mu \mathrm{M}$ proguanil, and stained with Hoechst or MitoTracker Red (10 nM). (D) Statistical analysis of MitoTracker signal for 40-50 total parasites from each condition in panel (C) from two independent experiments. MitoTracker signal was designated as focal if similar in size to nuclear signal (Hoechst stain) or dispersed if similar in size to the parasite cytoplasm (based on brightfield image). For clarity, error bars are not shown but standard errors of the mean were $\leq 10 \%$ in all cases. Cell percentage differences were analyzed by two-tailed unpaired t-test ( $p$ values in parentheses, ns $=$ not significant). Parasite counts for individual experiments are given in Figure 5-source data 1.

The online version of this article includes the following figure supplement(s) for figure 5 :

Source data 1. Raw parasite counts for individual microscopy experiments.

Figure supplement 1. Additional live microscopy images of mACP-aptamer/TetR-DOZI parasites.

atovaquone (Ke et al., 2011). We posited that it might be possible to rescue parasites with $d Q$ from mACP knockdown if the dominant cause of parasite death were ETC failure.

As a positive control and to provide a basis for comparison, we first confirmed that $15 \mu \mathrm{M}$ exogenous dQ substantially rescued parasites over multiple intraerythrocytic cycles from growth inhibition by $100 \mathrm{nM}$ atovaquone (Figure 5A), as previously reported (Ke et al., 2011). To test dQ rescue of parasites upon mACP knockdown, we synchronized the mACP-aptamer/TetR-DOZI parasites and monitored their growth $\pm \mathrm{aTc}$ and $\pm \mathrm{dQ}$ for several intraerythrocytic cycles. As before, -aTc parasites grew normally for 3 days but failed to expand into the third growth cycle on day 4 (Figure 5A). Addition of $d Q$ rescued parasite growth in the third growth cycle in -aTc conditions, similar to dQ rescue of parasites from atovaquone. However, in contrast to indefinite parasite rescue from atovaquone, 
$d \mathrm{Q}$ only rescued the third-cycle growth of parasites cultured -aTc, which failed to expand further and began to die off on day 6 in the fourth intraerythrocytic cycle (Figure $5 \mathrm{~A}$ ). On the basis of dQ rescue, we conclude that ETC failure is the immediate cause of parasite death upon mACP knockdown. However, the inability of $d Q$ to rescue parasites beyond the third cycle indicates that additional dysfunctions beyond the mitochondrial ETC contribute to parasite death on a longer timescale. These additional defects may involve mitochondrial fumarate hydratase and/or essential Fe-S cluster proteins in the cytoplasm and nucleus that depend on Nfs1 function (see 'Discussion' section).

To further unravel the effect of mACP knockdown on ETC function, we next tested if loss of mACP sensitized parasites to mitochondrial depolarization by proguanil. The ETC couples ubiquinone recycling to proton translocation from the mitochondrial matrix to the inner membrane space to establish a transmembrane electrochemical potential. Prior work has shown that parasites retain a second proton-pumping mechanism that also contributes to transmembrane potential and that is inhibited by proguanil (Painter et al., 2007; Skinner-Adams et al., 2019). Inhibition of ETC function kills parasites due to defective ubiquinone recycling but does not substantially depolarize mitochondria due to activity by this second, proguanil-sensitive pathway. However, ETC dysfunction plus proguanil treatment blocks both pathways for proton pumping and causes mitochondrial depolarization, which can be visualized by a failure to concentrate charged dyes like MitoTracker within the mitochondrion that leads to dispersed dye accumulation in the cytoplasm (Painter et al., 2007; Ke et al., 2018).

If mACP knockdown and subsequent loss of Rieske caused general ETC failure, we predicted that parasite treatment with sublethal proguanil would negate the ability of $d Q$ to rescue parasite growth from mACP knockdown in the third cycle and would cause mitochondrial depolarization. We repeated the prior growth assay $\pm \mathrm{aTc}$ and $\pm \mathrm{dQ}$ but also included $1 \mu \mathrm{M}$ proguanil. This proguanil concentration alone had no effect on parasite growth, as previously reported (Painter et al., 2007). However, when proguanil was combined with growth -aTc, dQ was unable to rescue parasite growth in the third cycle (Figure 5B). Microscopy analysis of parasites on day 3 of the growth assay revealed that proguanil treatment selectively prevented mitochondrial accumulation of MitoTracker Red in parasites grown -aTc, strongly suggesting mitochondrial depolarization (Figure 5C and D, Figure 5-figure supplement 1). This observation strongly supports the model that impaired ubiquinone recycling due to Rieske loss and ETC dysfunction is the immediate cause of parasite death upon mACP knockdown.

\section{Discussion}

The $P$. falciparum mitochondrion is a major antimalarial drug target, but nearly all organelle-specific inhibitors target cytochrome $b$ in ETC complex III or DHOD that depends on complex III function (Antonova-Koch et al., 2018; Goodman et al., 2017). Multiple metabolic pathways operate within the mitochondrion (van Dooren et al., 2006), but many are dispensable for blood-stage parasites, and few essential blood-stage functions beyond DHOD and ETC complexes III and IV have been identified. ISC biosynthesis is an ancient, essential mitochondrial function that has been well studied in yeast and mammalian cells but is sparsely studied in parasites (Gisselberg et al., 2013). We have identified a divergent and essential nexus between Fe-S cluster biogenesis and an evolutionary vestige of type II fatty acid synthesis in the P. falciparum mitochondrion.

\section{New molecular paradigm for essential ACP function without an acyl- Ppant group}

Most eukaryotic cells, including fungi, plants, and animals, retain a mitochondrial FASII pathway in which the acyl-ACP intermediate has been shown to critically mediate respiratory chain assembly, Fe-S cluster biogenesis, and ribosomal translation by binding to LYR-protein assembly factors (Majmudar et al., 2019; Angerer, 2015; Nowinski et al., 2018). These interactions and their dependence on ACP acylation have been proposed to constitute a regulatory feedback mechanism that couples the availability of acetyl-CoA for FASIl activity to respiratory chain assembly for oxidative phosphorylation and ATP synthesis (Van Vranken et al., 2018). Biochemical coupling of these pathways, however, has not been retained in Plasmodium, which has lost mitochondrial FASIl enzymes but retains a divergent ACP homolog incapable of 4-Ppant modification or acylation. Nevertheless, we have shown that $\mathrm{mACP}$ remains essential to parasites for Fe-S cluster biogenesis by binding to Isd11, which is the only LYR-protein homolog retained by P. falciparum. Isd11 binds to $\mathrm{mACP}$ via a novel interface molecular 
interface, and this interaction is critical stabilizing the mACP-Isd11-Nfs1 cysteine desulfurase complex. $P$. falciparum thus provides a new molecular paradigm for essential ACP function without an acylPpant group. This discovery emphasizes the ancient, fundamental role of ACP in mitochondrial Fe-S cluster biogenesis and suggests an evolutionary driving force to retain $\mathrm{mACP}$ interaction with the Isd11-Nfs1 complex-independent ACP's scaffolding role in fatty acid synthesis.

Prior in vitro work has provided evidence that activity of purified Isd11-Nfs1 does not strictly require association with ACP (Boniecki et al., 2017; Lill and Freibert, 2020). However, Nfs1 is unstable in its native mitochondrial context in the absence of $A C P$, as shown herein for $P$. falciparum and in prior studies of yeast (Van Vranken et al., 2016; Adam et al., 2006). Coupling Nfs1 stability (via Isd11) to ACP acylation in eukaryotes that retain mitochondrial FASIl has been proposed as a mechanism to up- and downregulate $\mathrm{Fe}-\mathrm{S}$ cluster biogenesis congruent with nutrient availability and cellular Fe-S cluster needs for respiration and growth (Lill and Freibert, 2020). This mechanism, however, cannot explain mitochondrial retention of an acylation-incompetent ACP homolog in Plasmodium. ACP may therefore play additional functional and/or regulatory roles that are essential for mitochondrial Fe-S cluster biogenesis and utilization in cells, perhaps involving metal-ion binding and sensing by ACP (e.g., iron or zinc) (Herrera et al., 2019; Qiu and Janson, 2004) or interactions with other mitochondrial networks akin to broad ACP interactions observed in E. coli (Gully et al., 2003). In this regard, it is interesting to note that anaerobic eukaryotic parasites like Giardia and Cryptosporidium lack a mitochondrion but retain a primitive mitosome for Fe-S cluster biogenesis that lacks ACP and Isd11 homologs in this compartment, where Nfs1 appears to function autonomously (Richards and van der Giezen, 2006; Braymer et al., 2021).

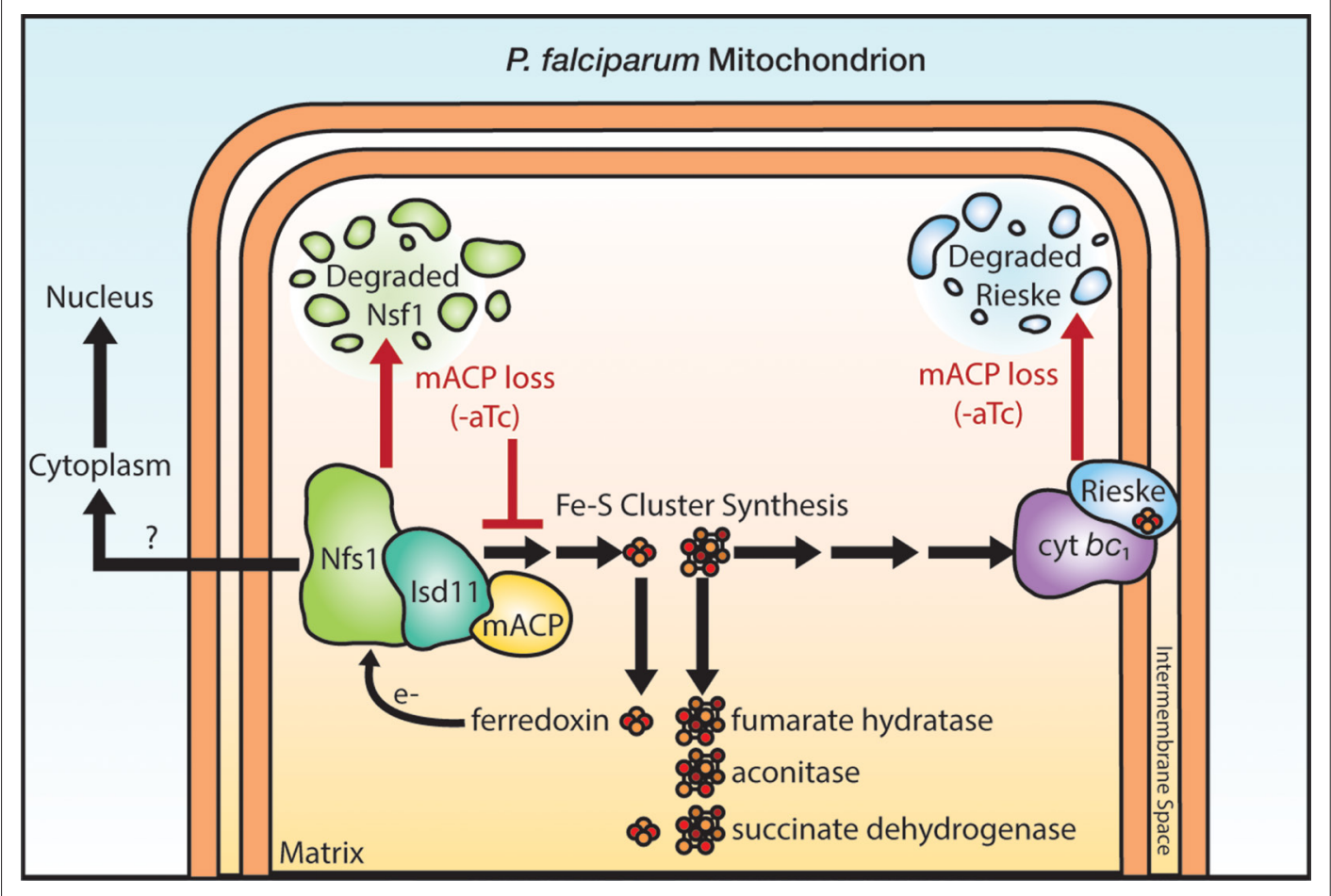

Figure 6. Schematic model for the impact of mACP knockdown (-aTc) on Fe-S cluster metabolism in P. falciparum parasites. The question mark indicates uncertainty in the functional role of mitochondrial Nfs 1 in supporting cytoplasmic Fe-S cluster biogenesis in parasites. For simplicity, the mACP/Isd11/Nfs1 complex is shown with only one monomer for each protein, rather than the functional dimer (Boniecki et al., 2017). Illustration by Megan Okada.

The online version of this article includes the following figure supplement(s) for figure 6:

Figure supplement 1. Sequence alignment of P. falciparum mACP (PF3D7_1208300) with homologs from T. gondii (TGME49_270310, e-value 2e-13), B. microti (BMR1_02g01455, e-value 7e-13), and V. brassicaformis (CEM12831, e-value $3 e-16)$. 


\section{Implications for parasites}

Loss of mitochondrial FASII, but retention of a Ppant-independent mACP lacking the conserved Ser, appears to be widespread among apicomplexan parasites, including Toxoplasma and Babesia (Figure 6-figure supplement 1). In the case of Toxoplasma gondii, BLAST analysis revealed two divergent mACP homologs (TGME49_270310 and TGME49_265538), differing in the presence of a Cys or Gly in place of the conserved Ser, in their transcriptional profiles, and in their predicted contribution to parasite infection of human fibroblasts (Sidik et al., 2016). Outside the phylum Apicomplexa, the only genomic evidence we found for a divergent ACP lacking the conserved Ser was in the related photosynthetic chromerid, Vitrella brassicaformis, but not in its algal cousin, Chromera velia (Figure 6-figure supplement 1). Thus, the adaptation of mitochondrial ACP to function without a Ppant group or acylation likely occurred on a similar evolutionary timeframe as the loss of plastid photosynthesis that accompanied the appearance of apicomplexan parasitism (Janouškovec et al., 2015).

Our study indicates that mitochondrial ACP function without an acyl-Ppant group is a parasitespecific adaptation that differentiates apicomplexan parasites from humans. Nevertheless, like yeast and human cells (Van Vranken et al., 2016; Majmudar et al., 2019), we observed that loss of ACP destabilizes Nfs1 to block Fe-S cluster assembly impairs the stability and function of the key Rieske subunit of ETC complex III and leads to parasite death (Figure 6). In yeast and mammalian cells, mACP plays a second role in Rieske maturation by binding to MZM1, an LYR-protein chaperone that stabilizes Rieske upon import into the mitochondrial matrix to receive its Fe-S cluster for subsequent insertion into ETC complex III by the AAA-ATPase, BCS1 (Majmudar et al., 2019; Atkinson et al., 2011). P. falciparum, however, appears to lack an MZM1 homolog (Figure 2-figure supplement 1), suggesting that parasites have evolved compensatory mechanisms for Rieske maturation in the absence of this ACP-dependent chaperone. In yeast, loss of mACP and/or the Rieske protein, which is a peripheral subunit inserted late in the assembly of ETC complex III, results in accumulation of a late core assembly intermediate of complex III that contains cyt b, cyt $c_{1}$, and other factors (Van Vranken et al., 2016; Atkinson et al., 2011). Our observation that cyt $c_{1}$ levels are retained upon loss of parasite mACP and Rieske in -aTc conditions (Figure 4) suggests that this late complex III assembly intermediate likely accumulates in P. falciparum similar to yeast. However, complex III assembly has been sparsely studied in Plasmodium and remains an important future challenge to understand.

Exogenous $\mathrm{dQ}$ rescued parasite growth upon $\mathrm{mACP}$ knockdown for one intraerythrocytic cycle but did not rescue growth on a longer timescale. This short-term rescue indicates that respiratory failure is the immediate cause of $P$. falciparum death upon loss of mACP (Painter et al., 2007; Ke et al., 2011). However, defects in essential processes beyond the mitochondrial ETC contribute to parasite death on longer timescales. These defects may involve mitochondrial fumarate hydratase ( $\mathrm{Ke}$ et al., 2015) and/or essential Fe-S cluster proteins in the cytosol and nucleus that depend on Nfs1 activity (Figure 6), including Rli1 required for cytoplasmic ribosome assembly and Rad3 and Pri2 that are essential for nuclear DNA metabolism (Dellibovi-Ragheb et al., 2013). In yeast, the mitochondrial transporter Atm 1 has been proposed to export a key sulfur-containing intermediate required for cytosolic Fe-S cluster assembly (Kispal et al., 1999; Braymer et al., 2021). In P. falciparum, the Mdr2 transporter (PF3D7_1447900) is 34 \% identical to yeast Atm1 and has been proposed to perform an analogous function in cytosolic Fe-S cluster assembly (van Dooren et al., 2006). However, Mdr2 appears to be dispensable for blood-stage parasites (van der Velden et al., 2015), and its possible mitochondrial-export role in cytoplasmic Fe-S cluster biogenesis remains untested. We also note prior studies in mammalian cells which suggest that cytoplasmic Fe-S cluster synthesis in these cells may be independent of mitochondrial Nfs1 activity (Maio and Rouault, 2020; Kim et al., 2018). Cytoplasmic Fe-S cluster metabolism in Plasmodium is sparsely studied, and its functional dependence on mitochondrial ISC proteins remains a key frontier to further test and understand.

In human cells, recent work has identified an LYR-motif protein (LOR8F8/MIEF1) that mediates interaction between $\mathrm{mACP}$ and mitochondrial ribosomes, suggesting a role for $\mathrm{mACP}$ in regulating mitochondrial translation (Brown et al., 2017; Rathore et al., 2018). Malaria parasites lack a homolog of LOR8F8 (Figure 2-figure supplement 1), and multiple experimental observations strongly suggest that $P$. falciparum mACP does not regulate mitochondrial translation. Prior work in yeast reported that loss of mitochondrial genome expression strongly reduces the stability of cyt $c_{1}$, due to loss of mitochondria-encoded cyt $b$ and destabilization of ETC complex III (Zara et al., 2004). Parasite cyt 


\section{Key resources table}

$b$ is also encoded on the mitochondrial genome. However, we observed that loss of mACP does not diminish the stability of parasite cyt $c_{1}$ (Figure 4 C). It was also recently shown that mitoribosome dysfunction in P. falciparum results in mitochondrial depolarization (Ke et al., 2018). In contrast to this phenotype, we observed that parasites retained their mitochondrial transmembrane potential upon mACP knockdown in -aTc conditions alone (Figure 5C). Collectively, these observations suggest that $\mathrm{mACP}$ function does not regulate mitochondrial translation in P. falciparum. Nevertheless, mACP may have additional functional interactions beyond the Isd11-Nfs1 complex that also contribute to its essentiality in parasites. Such interactions, however, will diverge from known interactions in yeast and humans that involve conserved LYR-motif proteins, and we have on-going studies to fully understand the functional interactome of $\mathrm{mACP}$ in the parasite mitochondrion.

The mitochondrial ETC is essential for malaria parasite viability in all lifecycle stages (Delves et al., 2012). The unique molecular features of $P$. falciparum mACP and its interaction with Isd11 that underpin essential functions of the ETC and broader Fe-S cluster utilization suggest the possibility of targeting this complex for antimalarial therapy. We have ongoing structural and biochemical studies to assess if parasite Isd11 retains a vestigial acyl-pantetheine binding pocket and to test if parasitespecific features of the mACP-Isd11 interaction can be selectively disrupted via small-molecule inhibitors that mimic the acyl-pantetheine group and/or broader hydrophobic features of this proteinprotein interface that are distinct from the human complex. On the basis of molecular conservation in other apicomplexan parasites, such inhibitors would likely function against other human pathogens such as Toxoplasma and Babesia.

\section{Materials and methods}

\begin{tabular}{|c|c|c|c|c|}
\hline $\begin{array}{l}\text { Reagent type } \\
\text { (species) or } \\
\text { resource }\end{array}$ & Designation & $\begin{array}{l}\text { Source or } \\
\text { reference }\end{array}$ & Identifiers & Additional information \\
\hline $\begin{array}{l}\text { Cell line } \\
\text { (Plasmodium } \\
\text { falciparum) }\end{array}$ & $\mathrm{Dd} 2$ & PMID:1970614 & $\begin{array}{l}\text { BEl Resources } \\
\text { MRA-156 }\end{array}$ & \\
\hline $\begin{array}{l}\text { Cell line } \\
\text { (Plasmodium } \\
\text { falciparum) }\end{array}$ & $\begin{array}{l}\text { Dd2 mACP-HA-FLAG 10X } \\
\text { Aptamer/TetR-DOZI }\end{array}$ & Made for this study & & $\begin{array}{l}\text { Described in Materials and methods. Can be } \\
\text { obtained from Sigala lab. }\end{array}$ \\
\hline $\begin{array}{l}\text { Cell line } \\
\text { (Plasmodium } \\
\text { falciparum) }\end{array}$ & $\begin{array}{l}\text { Dd2 } \\
\text { Cyt } c_{1}-\text { GFP (pTEOE) mACP-HA- } \\
\text { FLAG 10X Aptamer/TetR-DOZI }\end{array}$ & Made for this study & & $\begin{array}{l}\text { Described in Materials and methods. Can be } \\
\text { obtained from Sigala lab. }\end{array}$ \\
\hline $\begin{array}{l}\text { Cell line } \\
\text { (Plasmodium } \\
\text { falciparum) }\end{array}$ & $\begin{array}{l}\text { Dd2 } \\
\text { Rieske-HA }(p T E O E) \text { mACP-HA- } \\
\text { FLAG 10X Aptamer/TetR-DOZI }\end{array}$ & Made for this study & & $\begin{array}{l}\text { Described in Materials and methods. Can be } \\
\text { obtained from Sigala lab. }\end{array}$ \\
\hline $\begin{array}{l}\text { Cell line } \\
\text { (Plasmodium } \\
\text { falciparum) }\end{array}$ & $\begin{array}{l}\text { Dd2 mACP-HA }(\mathrm{pTEOE}) \text { mACP- } \\
\text { HA-FLAG 10X Aptamer/TetR- } \\
\text { DOZI }\end{array}$ & Made for this study & & $\begin{array}{l}\text { Described in Materials and methods. Can be } \\
\text { obtained from Sigala lab. }\end{array}$ \\
\hline $\begin{array}{l}\text { Cell line } \\
\text { (Plasmodium } \\
\text { falciparum) }\end{array}$ & $\begin{array}{l}\text { Dd2 } \\
\text { F113A mACP-HA } 2 \text { (pTEOE) } \\
\text { mACP-HA-FLAG 10X Aptamer/ } \\
\text { TetR-DOZI }\end{array}$ & Made for this study & & $\begin{array}{l}\text { Described in Materials and methods. Can be } \\
\text { obtained from Sigala lab. }\end{array}$ \\
\hline $\begin{array}{l}\text { Cell line } \\
\text { (Plasmodium } \\
\text { falciparum) }\end{array}$ & Dd2 mACP-HA $A_{2}$ (pTEOE) & Made for this study & & $\begin{array}{l}\text { Described in Materials and methods. Can be } \\
\text { obtained from Sigala lab. }\end{array}$ \\
\hline $\begin{array}{l}\text { Cell line } \\
\text { (Plasmodium } \\
\text { falciparum) }\end{array}$ & $\mathrm{Dd} 2 \mathrm{aACP}-\mathrm{HA}_{2}$ (pTEOE) & Made for this study & & $\begin{array}{l}\text { Described in Materials and methods. Can be } \\
\text { obtained from Sigala lab. }\end{array}$ \\
\hline $\begin{array}{l}\text { Cell line } \\
\text { (Plasmodium } \\
\text { falciparum) }\end{array}$ & $\begin{array}{l}\text { Dd2 mACP-HA } 2 \text { (pTEOE) } \\
\text { Isd11-GFP (pRL2) }\end{array}$ & Made for this study & & $\begin{array}{l}\text { Described in Materials and methods. Can be } \\
\text { obtained from Sigala lab. }\end{array}$ \\
\hline $\begin{array}{l}\text { Cell line } \\
\text { (Escherichia coli) }\end{array}$ & $\begin{array}{l}\text { BL21/DE3 } \\
\text { mACP-HA }_{2}(p E T 28 a)\end{array}$ & Made for this study & & $\begin{array}{l}\text { Described in Materials and methods. Can be } \\
\text { obtained from Sigala lab. }\end{array}$ \\
\hline
\end{tabular}

Continued on next page 
Continued

\begin{tabular}{|c|c|c|c|c|}
\hline $\begin{array}{l}\text { Reagent type } \\
\text { (species) or } \\
\text { resource }\end{array}$ & Designation & $\begin{array}{l}\text { Source or } \\
\text { reference }\end{array}$ & Identifiers & Additional information \\
\hline $\begin{array}{l}\text { Cell line } \\
\text { (Escherichia coli) }\end{array}$ & $\begin{array}{l}\text { BL21/DE3 } \\
\triangle 2-51 \text { mACP-HA } 2 \text { (pET28a) }\end{array}$ & Made for this study & & $\begin{array}{l}\text { Described in Materials and methods. Can be } \\
\text { obtained from Sigala lab. }\end{array}$ \\
\hline $\begin{array}{l}\text { Cell line } \\
\text { (Escherichia coli) }\end{array}$ & $\begin{array}{l}\text { BL21/DE3 } \\
\text { Isd11-His }(p E T 21 d) m A C P-H A_{2} \\
\text { (pET28a) }\end{array}$ & Made for this study & & $\begin{array}{l}\text { Described in Materials and methods. Can be } \\
\text { obtained from Sigala lab. }\end{array}$ \\
\hline $\begin{array}{l}\text { Cell line } \\
\text { (Escherichia coli) }\end{array}$ & $\begin{array}{l}\text { BL21/DE3 } \\
\text { LYR12-14AAA } \\
\text { Isd11-His }(p E T 21 d) m A C P-H A_{2} \\
\text { (pET28a) }\end{array}$ & Made for this study & & $\begin{array}{l}\text { Described in Materials and methods. Can be } \\
\text { obtained from Sigala lab. }\end{array}$ \\
\hline $\begin{array}{l}\text { Cell line } \\
\text { (Escherichia coli) }\end{array}$ & $\begin{array}{l}\text { BL21/DE3 } \\
\text { Isd11-His }(p E T 21 d) \\
\Delta 2-51 \text { mACP-HA } 2 \text { (pET28a) }\end{array}$ & Made for this study & & $\begin{array}{l}\text { Described in Materials and methods. Can be } \\
\text { obtained from Sigala lab. }\end{array}$ \\
\hline $\begin{array}{l}\text { Cell line } \\
\text { (Escherichia coli) }\end{array}$ & $\begin{array}{l}\text { BL21/DE3 } \\
\text { Isd11-His }(p E T 21 d) \\
\text { F113A }(\Delta 2-51) \text { mACP-HA } \\
\text { (pET28a) }\end{array}$ & Made for this study & & $\begin{array}{l}\text { Described in Materials and methods. Can be } \\
\text { obtained from Sigala lab. }\end{array}$ \\
\hline $\begin{array}{l}\text { Cell line } \\
\text { (Escherichia coli) }\end{array}$ & $\begin{array}{l}\text { BL21/DE3 } \\
\text { Isd11-His }(p E T 21 d) \\
\triangle 2-40 \text { aACP-HA } 2 \text { (pET28a) }\end{array}$ & Made for this study & & $\begin{array}{l}\text { Described in Materials and methods. Can be } \\
\text { obtained from Sigala lab. }\end{array}$ \\
\hline $\begin{array}{l}\text { Chemical } \\
\text { compound, drug }\end{array}$ & Anhydrotetracycline & Caymen Chemicals & $\begin{array}{l}\text { Cat. no. } \\
10009542\end{array}$ & \\
\hline $\begin{array}{l}\text { Chemical } \\
\text { compound, drug }\end{array}$ & Atovaquone & Caymen Chemicals & $\begin{array}{l}\text { Cat. no. } \\
95233184\end{array}$ & \\
\hline $\begin{array}{l}\text { Chemical } \\
\text { compound, drug }\end{array}$ & Decyl-ubiquinone & Caymen Chemicals & $\begin{array}{l}\text { Cat. no. } \\
55486005\end{array}$ & \\
\hline $\begin{array}{l}\text { Chemical } \\
\text { compound, drug }\end{array}$ & Proguanil & Sigma-Aldrich & $\begin{array}{l}\text { Cat. no. } \\
637,321\end{array}$ & \\
\hline Antibody & $\begin{array}{l}\text { Anti-HA } \\
\text { (rat monoclonal 3F10) }\end{array}$ & Sigma-Aldrich & $\begin{array}{l}\text { Cat. no. } \\
\text { 11867423001, } \\
\text { RRID:AB_390918 }\end{array}$ & $(1: 1000)$ \\
\hline Antibody & $\begin{array}{l}\text { Anti-HSP60 } \\
\text { (rabbit polyclonal) }\end{array}$ & Novus & $\begin{array}{l}\text { Cat. no. } \\
\text { NBP2-12734 }\end{array}$ & $(1: 1000)$ \\
\hline Antibody & $\begin{array}{l}\text { Anti-Nfs } 1 \\
\text { (rabbit polyclonal) }\end{array}$ & Abcam & Cat. no. ab229829 & $(1: 1000)$ \\
\hline Antibody & $\begin{array}{l}\text { Anti-GFP } \\
\text { (goat polyclonal) }\end{array}$ & Abcam & $\begin{array}{l}\text { Cat. no. ab5450, } \\
\text { RRID:AB_304897 }\end{array}$ & $(1: 1000)$ \\
\hline Antibody & $\begin{array}{l}\text { Anti-EF1 } \alpha \\
\text { (rabbit polyclonal) }\end{array}$ & PMID:11251817 & & $(1: 1000)$ \\
\hline Antibody & $\begin{array}{l}\text { Anti-EF1 } \beta \\
\text { (rabbit polyclonal) }\end{array}$ & PMID:11251817 & & $(1: 1000)$ \\
\hline Antibody & $\begin{array}{l}\text { Anti-His }- \text { DyLight680 } \\
\text { (mouse monoclonal) }\end{array}$ & Thermo Fisher & $\begin{array}{l}\text { Cat. no. } \\
\text { MA121315D680, } \\
\text { RRID:AB_2536987 }\end{array}$ & $(1: 1000)$ \\
\hline
\end{tabular}

\section{Cloning}

For episomal protein expression, the genes encoding mitochondrial ACP (PF3D7_1208300), Rieske protein (PF3D7_1439400), cytochrome c (PF3D7_ 1462700), and apicoplast ACP (PF3D7_0208500) were PCR-amplified from Dd2 parasite cDNA using primer sets 1/2,3/4, and 5/6, and 27/28 (Supplementary file 1), respectively, and cloned into PTEOE (Sigala et al., 2015) at the Xhol/Avrll sites in frame with a C-terminal dual hemagglutinin $\left(\mathrm{HA}_{2}\right)$ tag (mACP and Rieske) or GFP tag (cytochrome $c_{1}$ ). The PTEOE vector contains the HSP86 promoter to drive episomal protein expression, encodes human DHFR as a positive selection cassette (Sigala et al., 2015), and is co-transfected with plasmid 
pHTH that contains the piggyBac transposase (Balu et alo, 2005) for integration into the parasite genome. Ligation-independent cloning was performed with the QuantaBio RepliQa HiFi Assembly Mix (VWR 95190-050). Cloning reaction mixes were transformed into Top10 chemically competent cells, and bacterial clones were selected for carbenicillin (Sigma C3416) resistance. Plasmid DNA was isolated using the PureLink Plasmid Miniprep system (Invitrogen K210011), and correct plasmid insert sequences were confirmed by Sanger sequencing (University of Utah DNA Sequencing Core) using vector-specific primers.

For recombinant protein expression in E. coli, the gene for P. falciparum Nfs1(PF3D7_0727200) was cloned from Dd2 parasite $c D N A$, and genes for $\mathrm{mACP}$, truncated $\mathrm{mACP}(\triangle 2-50)$, and truncated aACP $(\triangle 2-40)$ were subcloned by PCR from the PTEOE plasmid using primer pairs $7 / 8,9 / 10,11 / 12$, and 29/30 (Supplementary file 1), respectively. These genes were inserted into the $\mathrm{Ncol} / \mathrm{Xhol}$ sites of pET28a (Novagen 69864) with a C-terminal $\mathrm{HA}_{2}$ tag using ligation-independent cloning with the QuantaBio system. P. falciparum Isd11 was PCR-amplified from Dd2 parasite cDNA using primer sets $13 / 14$ and cloned into the Ncol/Xhol sites of pET21d (Novagen/MilliporeSigma 69743) in-frame with the $\mathrm{C}$-terminal $\mathrm{His}_{6}$ tag using ligation-independent methods. Correct insert sequences were verified by Sanger sequencing of purified plasmid DNA.

\section{CRISPR-Cas9 genome editing}

CRISPR/Cas9-stimulated repair by double-crossover homologous recombination was used to tag the mACP gene to encode a C-terminal HA-FLAG epitope fusion tag and the 3' 10X aptamer/TetR-DOZI system (Ganesan et al., 2016) to enable regulated mACP expression using anhydrotetracycline (aTc, Caymen Chemicals 10009542). A guide RNA sequence corresponding to TGGTATTGTTATATTAAATT was cloned with ligation-independent methods using primer pair 15/16 (Supplementary file 1) into a modified version of the previously published pAIO CRISPR/Cas9 vector (Spillman et al., 2017) in which the BtgZl site was replaced with a unique Hindlll site to facilitate cloning. To tag the mACP gene, the donor pMG75 repair plasmid was created by using ligation-independent cloning to insert a gBlock gene fragment ordered from IDT into the unique Ascl/Aatll cloning sites. This gBlock contained $200 \mathrm{bp}$ of the $3^{\prime}$ untranslated region of the mACP gene (starting at position 135 downstream from the TAA stop codon), an Afel site, and the $312 \mathrm{bp}$ of the $3^{\prime}$ end of the mACP coding sequence (excluding the 129 bp intron). The gBlock sequence included a shield mutation to ablate the CRISPR PAM sequence CGG that immediately follows the gRNA sequence above in the antisense strand of the coding sequence by mutating it to CTG, resulting in a silent mutation of the Ser 125 codon from TCC to TCT. Before transfection, the PMG75 vector was linearized by Afel digestion performed overnight at $37^{\circ} \mathrm{C}$, followed by deactivation with Antarctic Phosphatase (NEB M0289S).

\section{Site-directed mutagenesis}

The mACP Phe113Ala (pTEOE) and Isd11 LYR12-14AAA (pET21d) mutations were introduced by PCR using primer pairs $17 / 18$ and $19 / 20$, respectively. For mACP, primer pairs $9 / 18$ and $17 / 10$ were used to introduce the Phe113Ala mutation and generate two insert fragments by PCR using the MACPpET28a plasmid as template. The two fragments were joined by overlap sewing PCR using primer set 9/10. The same PCR process and primers pairs $13 / 20$ and 19/14 were used to make Isd11 LYR1214AAA using Isd11-pET21d as the template. Primer pairs 24/14, 25/14, and 26/14 were used to make the respective single Isd11 LYR mutations of L12A, Y13A, and R14A. Correct insert sequences for the mutated genes were confirmed by Sanger sequencing of plasmid DNA. Primer sequences are listed in Supplementary file 1.

\section{Parasite culturing and transfection}

All experiments were performed using P. falciparum Dd2 parasites (Wellems et alo, 1990), whose identity was confirmed based on expected drug resistance. Parasite cultures were mycoplasma-free by PCR test. Parasite culturing was performed in Roswell Park Memorial Institute medium (RPMI1640, Thermo Fisher 23400021) supplemented with $2.5 \mathrm{~g} / \mathrm{L}$ Albumax I Lipid-Rich BSA (Thermo Fisher 11020039), $15 \mathrm{mg} / \mathrm{L}$ hypoxanthine (Sigma H9636), $110 \mathrm{mg} / \mathrm{L}$ sodium pyruvate (Sigma P5280), 1.19 g/L HEPES (Sigma H4034), $2.52 \mathrm{~g} / \mathrm{L}$ sodium bicarbonate (Sigma S5761), $2 \mathrm{~g} / \mathrm{L}$ glucose (Sigma G7021), and $10 \mathrm{mg} / \mathrm{L}$ gentamicin (Invitrogen Life Technologies 15750060). Cultures were maintained at $2 \%$ 
hematocrit in human erythrocytes obtained from the University of Utah Hospital blood bank, at $37^{\circ} \mathrm{C}$, and $5 \% \mathrm{CO}_{2}$.

For episomal protein expression using the PTEOE vector, parasite-infected erythrocytes were transfected in $1 \times$ cytomix containing $50-100 \mu \mathrm{g}$ of purified plasmids and $25 \mu \mathrm{g}$ of the pHTH transposase plasmid by electroporation in $0.2 \mathrm{~cm}$ cuvettes using a Bio-Rad Gene Pulser Xcell system (0.31 $\mathrm{kV}, 925 \mu \mathrm{F})$. Transfected cultures were allowed to expand in the absence of drug for $48 \mathrm{hr}$ and then selected in 5 nM WR99210 (Jacobus Pharmaceuticals). Stable drug-resistant parasites returned from transfection in 2-8 weeks. The pRL2 plasmid encoding Isd11-GFP (Gisselberg et al., 2013) was a gift from Sean Prigge (Johns Hopkins University). This plasmid was transfected into stably selected mACP$\mathrm{HA}_{2}$ (pTEOE) Dd2 parasites and selected with $5 \mathrm{nM}$ WR99210 and $6 \mu \mathrm{M}$ blasticidin-S (ThermoFisher/ Gibco R21001). Similarly, pTEOE plasmids for Rieske-HA $A_{2}$ cyt $c_{1}-G F P$, and WT or Phe113Ala mACP$\mathrm{HA}_{2}$ were separately transfected into the polyclonal Dd2 mACP-HA-FLAG aptamer/TetR-DOZI knockdown line (described below) and selected with $5 \mathrm{nM}$ WR99210, $6 \mu \mathrm{M}$ blasticidin-S, and $0.5 \mu \mathrm{M}$ aTc.

For CRISPR/Cas9-based editing of the mACP genomic locus, 50-100 $\mu \mathrm{g}$ each of the linearized pMG75 donor plasmid and pAIO plasmid (expressing gRNA-Cas9) were mixed and transfected into Dd2 parasites and selected with blasticidin-S in the presence of 0.5-1 $\mu \mathrm{M}$ aTc. Polyclonal parasites returning from transfection were genotyped by PCR using the primer sets 21/22 and 1/22 (Supplementary file 1) for the wild-type mACP gene. Primer sets $21 / 23$ and $1 / 23$ were used to test parasites for integration and tagging of the mACP gene. This analysis indicated that DNA recombination with the $5^{\prime}$ homology arm occurred upstream of the single intron in the WT genomic locus, resulting in the edited gene lacking this intron. A PCR amplicon for the edited but not WT (unmodified) mACP gene was detected in polyclonal parasites, which were used for most subsequent experiments. Clonal parasite integrants were isolated by limiting dilution of the polyclonal culture and were PCR-genotyped using the same primer sets as above. All growth assays involving conditional mACP expression were performed with polyclonal parasites except for $\mathrm{dQ}$ rescue experiments in Figure 5, which were performed with clone B5.

\section{Parasite growth assays}

Parasites were synchronized to rings by treatment with $5 \%$ D-sorbitol. For growth assays involving regulated $\mathrm{mACP}$ expression, aptamer-tagged parasites were washed three times after synchronization with RPMI media lacking aTc and then divided into two equal parts before supplementing one part with $0.5 \mu \mathrm{M}$ aTc. Growth was monitored by diluting sorbitol-synchronized parasites to $\sim 0.5 \%$ parasitemia and allowing culture expansion over several days with daily media changes. For $d Q$ (Caymen Chemicals 55486005) rescue experiments, dQ was dissolved in DMSO and added directly to cultures with a final concentration of $15 \mu \mathrm{M}$ and $\leq 0.3 \%$ DMSO. Proguanil (Sigma 637321) was added to cultures at a final concentration of $1 \mu \mathrm{M}$ at the beginning of growth experiments (Figure $5 B$ ) and added at $5 \mu \mathrm{M}$ at the end of the first cycle (24 hr after synchronization) in mitochondrial depolarization studies (Figure 5C). Atovaquone (Caymen Chemicals 95233184) was used as a positive control for dQ-rescue and membrane-polarization experiments and was dissolved in DMSO and added to corresponding cultures at a final concentration of $100 \mathrm{nM}$ and $\leq 0.3 \%$ DMSO. Parasitemia was monitored daily by flow cytometry by diluting $10 \mu \mathrm{L}$ of each parasite culture well into $200 \mu \mathrm{L}$ of $1.0 \mu \mathrm{g} / \mathrm{mL}$ acridine orange (Invitrogen Life Technologies A3568) in phosphate buffered saline (PBS) and analysis on a BD FACSCelesta system monitoring SSC-A, FSC-A, PE-A, FITC-A, and PerCP-Cy5-5-A channels. Parasitemia was determined by flow cytometry in $\geq 2$ biological replicates (distinct parasite samples set up in parallel) and reported as an average and standard deviation, as indicated in each figure legend. Graphs were plotted using GraphPad Prism 9.0.

\section{Immunoprecipitation experiments}

Dd2 parasites expressing mACP-HA $\mathrm{H}_{2}$ or endogenously tagged mACP-HA/FLAG were harvested by centrifugation, treated with $0.05 \%$ saponin (Sigma 84510) in PBS for $5 \mathrm{~min}$ at room temperature to lyse erythrocytes, and spun down by centrifugation at $4000 \mathrm{rpm}$ for $30 \mathrm{~min}$ at $4^{\circ} \mathrm{C}$. For HA-tagged proteins, IP was performed using Pierce anti-HA magnetic beads (Thermo Scientific 88836). Parasite pellets from $\sim 50 \mathrm{~mL}$ cultures were lysed in $1 \mathrm{~mL} \mathrm{1 \%}$ Triton (Sigma 9002931) or $1 \%$ digitonin in cold $1 \times$ PBS plus protease inhibitor (Thermo Scientific A32955). Pellets were dispersed by brief sonication on a Branson sonicator equipped with a microtip probe and then incubated at $4^{\circ} \mathrm{C}$ for $1 \mathrm{hr}$ on a rotator. 
Lysates were clarified by centrifugation at 13,000 rpm for $10 \mathrm{~min} .30 \mu \mathrm{L}$ of anti-HA magnetic beads was equilibrated in $170 \mu \mathrm{L}$ cold $1 \times$ Tris-buffered saline $(20 \mathrm{mM}$ Tris, $\mathrm{pH} 7.6,150 \mathrm{mM} \mathrm{NaCl})+0.05 \%$ Tween-20 (TBS-T), and beads were collected against a magnetic stand. Parasite lysates supernatants $(1 \mathrm{~mL})$ were added to the equilibrated beads and incubated for $1 \mathrm{hr}$ at $4^{\circ} \mathrm{C}$ by rotation. Beads were washed three times with ice-cold $1 \times$ TBS-T. Bound proteins were eluted with $\sim 100 \mu \mathrm{L} 8 \mathrm{M}$ urea (in $100 \mathrm{mM}$ Tris at $\mathrm{pH} 8.8$ ) and stored at $-20^{\circ} \mathrm{C}$ until use.

Parasite samples expressing GFP-tagged proteins (Isd11-GFP and cyt $c_{1}$-GFP) were harvested and lysed as above. IP of GFP-tagged proteins was performed using $50 \mu \mathrm{L}$ of protein A or G Dynabeads (Thermo Scientific 10001D or 10003D) that were equilibrated as described above. $150 \mu \mathrm{L}$ of cold $1 \times$ TBS-T plus $0.5 \mu \mathrm{L}$ of goat anti-GFP primary antibody (Abcam ab5450) was added to the equilibrated beads and incubated for $10 \mathrm{~min}$ before adding the clarified lysate as described above. Washes and elutions were performed as described above.

For MS experiments, IP eluates were precipitated by adding $100 \%$ trichloroacetic acid (Sigma 76039 ) to a final concentration of $20 \%$ and incubated on ice for $1 \mathrm{hr}$ in $1.6 \mathrm{~mL}$ Eppendorf tubes. Tubes were spun at $13,000 \mathrm{rpm}$ for $25 \mathrm{~min}$ at $4^{\circ} \mathrm{C}$. Supernatants were removed by vacuum aspiration, and protein pellets were washed once with $500 \mu \mathrm{L}$ of cold acetone. The protein pellets were air-dried for $30 \mathrm{~min}$ and stored at $-20^{\circ} \mathrm{C}$.

\section{Western blot analyses}

Samples were fractionated by SDS-PAGE using 10\% acrylamide gels run at $120 \mathrm{~V}$ in the Bio-Rad mini-PROTEAN electrophoresis system. For SDS-PAGE analysis of whole parasite extracts, $1 \times$ sample buffer containing beta-mercaptoethanol was added to parasite samples before heating at $95^{\circ} \mathrm{C}$ for $10 \mathrm{~min}$ and centrifuging at 13,000 rpm for $5 \mathrm{~min}$. Fractionated proteins were transferred from polyacrylamide gel to nitrocellulose membrane at $100 \mathrm{~V}$ for $1 \mathrm{hr}$ using the Bio-Rad wet-transfer system. Membranes were blocked in 1\% casein/PBS for $1 \mathrm{hr}$ at room temperature and then probed with primary antibody overnight at $4^{\circ} \mathrm{C}$ and secondary antibody at room temperature for $1 \mathrm{hr}$. Samples containing HA-tagged proteins were probed with a 1:1000 dilution of Roche rat anti-HA monoclonal 3F10 primary (Sigma 11867423001) and a 1:5000 dilution of donkey anti-rat DyLight800 (Invitrogen Life Technologies SA5-10032). Membranes containing GFP-tagged proteins were probed with a 1:1000 of goat anti-GFP polyclonal antibody (Abcam ab5450) and a 1:5000 dilution of donkey anti-rabbit DyLight680 (Invitrogen SA5-10042). Membranes were probed for P. falciparum Nfs1 with a 1:1000 dilution of rabbit anti-Nfs1 (Abcam ab229829) 1:5000 dilution of donkey anti-rabbit DyLight800 (Invitrogen SA5-10044). Rabbit antibodies that recognize P. falciparum HSP60 (Novus NBP2-12734) or elongation factor alpha or beta (EF1 $\alpha$ or EF1 $\beta$ ) (Mamoun and Goldberg, 2001) were used as loading controls at 1:1000 dilution. Membranes were probed with mouse monoclonal anti-His 6 -DyLight680 (Invitrogen MA121315D680) at 1:1000 dilution. Membranes were imaged using the Licor Odyssey system. All image adjustments, including contrast and brightness, were linear. WBs were repeated 2-3 times with independent samples. Uncropped images for all WBs are shown in source data.

WBs involving mACP knockdown were analyzed by densitometry using ImageJ (version 2.1.0/1.53c). The integrated signal intensity for each protein of interest (Nfs1, Rieske-HA $\mathrm{H}_{2} \mathrm{HSP} 60$, or cyt $c_{1}$-GFP) was normalized to that of the loading control (cytosolic EF1 $\alpha$ or EF1 $\beta$ ) in the same vertical lane for each experiment. The relative signal intensities (protein of interest/loading control) from 2 to 3 replicate experiments were used to calculate a mean \pm SEM and normalized to + aTc conditions for each protein. Differences in normalized signal intensities \pm aTc were analyzed by two-tailed unpaired t-test in GraphPad Prism 9.0.

\section{Mass spectrometry of parasite IP samples}

For identification of $\mathrm{mACP}$-interacting proteins in IP experiments, protein samples were reduced and alkylated using $5 \mathrm{mM}$ Tris (2-carboxyethyl) phosphine and $10 \mathrm{mM}$ iodoacetamide, respectively, and then enzymatically digested by sequential addition of trypsin and lys- $C$ proteases, as previously described (Florens et al., 2006; Wohlschlegel, 2009). The digested peptides were desalted using Pierce C18 tips (Thermo Fisher Scientific), dried, and resuspended in 5\% formic acid. Approximately $1 \mu \mathrm{g}$ of digested peptides was loaded onto a 25-cm-long, $75 \mu \mathrm{m}$ inner diameter fused silica capillary packed in-house with bulk C18 reversed phase resin (1.9 $\mathrm{m}, 100 \mathrm{~A}$ pores, Dr. Maisch GmbH). The $140 \mathrm{~min}$ water-acetonitrile gradient was delivered using a Dionex Ultimate 3000 ultra high-performance liquid 
chromatography system (Thermo Fisher Scientific) at a flow rate of $200 \mathrm{~nL} / \mathrm{min}$ (Buffer A: water with $3 \%$ DMSO and $0.1 \%$ formic acid; Buffer B: acetonitrile with $3 \%$ DMSO and $0.1 \%$ formic acid). Eluted peptides were ionized by the application of distal $2.2 \mathrm{kV}$ and introduced into the Orbitrap Fusion Lumos mass spectrometer (Thermo Fisher Scientific) and analyzed by tandem mass spectrometry. Data was acquired using a Data-Dependent Acquisition method consisting of a full MS1 scan (resolution $=120,000$ ) followed by sequential MS2 scans (resolution $=15,000$ ) to utilize the remainder of the 3 s cycle time. Data analysis was accomplished using the Integrated Proteomics Pipeline 2 (Integrated Proteomics Applications, San Diego, CA). Data was searched against the protein database from P. falciparum 3D7 downloaded from UniprotKB (10,826 entries) in October 2013. Tandem MS spectra searched using the ProLuCID algorithm followed by filtering of peptide-to-spectrum matches by DTASelect using a decoy database-estimated false discovery rate of $<1 \%$.

To analyze specific enrichment of Nfs1 in the mACP-HA IP versus aACP-HA IP for each matched experiment, the spectral counts observed for each protein in the mACP IP were divided by the spectral counts observed for the same protein in the aACP IP. For purposes of this analysis, all zero values for spectral counts were arbitrarily converted to a 1 . The $\log _{2}$ value of the mACP/aACP spectral count ratio for each protein was plotted for each of the three matched experiments. Nfs 1 was highly enriched in the mACP data set and among the top $0.5-1 \%$ of proteins based on spectral count ratio.

\section{Mass spectrometry of purified recombinant protein samples}

For experiments involving Ni-NTA pulldown of recombinant $P$. falciparum Isd11-His 6 from $E$. coli coexpressing $P$. falciparum $(\triangle 2-50)$ mACP (described below), purified proteins were identified by proteolytic digestion and tandem mass spectrometry. Proteins were reduced with DTT for $45 \mathrm{~min}$ at $60^{\circ} \mathrm{C}$ and then alkylated with iodoacetamide for $30 \mathrm{~min}$ at room temperature. Proteins were digested overnight at $38^{\circ} \mathrm{C}$ with Trypsin/LysC mixture using $1 \mu \mathrm{g}$ of trypsin per sample and quenched by acidification with $1 \%$ formic acid to a $\mathrm{pH}$ of 2-3. Reversed-phase nano-LC/MS/MS was performed on an UltiMate 3000 RSLCnano system (Dionex) coupled to a ThermoScientific QExactive-HF mass spectrometer equipped with a nanoelectrospray source. Concentrated samples were diluted with a $1: 1$ ratio of sample:0.1\% formic acid in water. $5 \mu \mathrm{L}$ of the samples were injected onto the liquid chromatograph. A gradient of reversed-phase buffers (Buffer A: $0.2 \%$ formic acid in water; Buffer B: $0.2 \%$ formic acid in acetonitrile) at a flow rate of $150 \mu \mathrm{L} / \mathrm{min}$ at $60^{\circ} \mathrm{C}$ was setup. The LC run lasted for 83 min with a starting concentration of $5 \%$ buffer B increasing to $55 \%$ over the initial $53 \mathrm{~min}$ and a further increase in concentration to $95 \%$ over $63 \mathrm{~min}$. A 40-cm long/100 $\mu \mathrm{m}$ inner diameter nanocolumn was employed for chromatographic separation. The column is a reverse-phase BEH C18 $3.0 \mu \mathrm{m}$ nanocolumn. MS/ MS data was acquired using an auto-MS/MS method selecting the most abundant precursor ions for fragmentation. The mass-to-charge range was set to 350-1800. Mascot generic format (MGF) files were generated from the raw MS/MS data. Mascot (version 2.6) uses the MGF file for database searching and protein identification. For these samples, the Custom database was searched with the Plasmodium taxonomy selected. The parameters used for the Mascot searches were trypsin digest; two missed cleavages; carbamidomethylation of cysteine set as fixed modification; oxidation of methionine and acetylation of the $\mathrm{n}$-terminus were set as variable modifications; and the maximum allowed mass deviation was set at $11 \mathrm{ppm}$.

\section{Sequence homology searches}

Sequence similarity searches of the $P$. falciparum genome for protein homologs of known LYR proteins from yeast and humans (Angerer, 2015) were performed by BLASTP analysis as implemented at the Plasmodium Genomics Resource webpage (https://www.plasmodb.org, release 48). The amino acid sequence of each human LYR protein homolog was used as bait, based on the following Uniprot accession codes: LYRM1 (043325), LYRM2 (Q9NU23), LYRM3 (Q9Y6M9), LYRM4 (Q9HD34), LYRM5 (Q6IPRI), LYRM6 (P56556), LYRM7 (Q5U5X 0), LYRM8 (A6NFY7), LYRM9 (A8MSI8), ACN9 (Q9NRP4), C7orf55 (Q96HJ9), and LOR8F8. Only protein hits with e-values $<0.01$ were retained.

To identify divergent ACP homologs in other organisms that also lacked the conserved Ser, the amino acid sequence for $P$. falciparum mACP starting with Leu51 was submitted as query to $\mathrm{NCBI}$ BLASTP (e-value threshold of 0.05). This search identified multiple homologs within the phylum Apicomplexa that also lacked the conserved Ser (e.g., T. gondii TGME49_270310 and Babesia microti BMR1_02g0145). To identify homologs outside of Apicomplexa, this search was repeated with 
Apicomplexa as an exclusion criterion. A single protein sequence from V. brassicaformis (CEM21745, e-value of $3 e-16)$ was the only ACP homolog identified in this expanded search that lacked the conserved Ser. An alignment of these homologs with $P$. falciparum $m A C P$ is shown in Figure 6-figure supplement 1.

\section{Fluorescence microscopy}

For live-cell experiments, parasite nuclei were visualized by incubating samples with $1-2 \mu \mathrm{g} / \mathrm{mL}$ Hoechst 33342 (Thermo Scientific Pierce 62249) for 10-20 min at room temperature. The parasite mitochondrion was visualized by incubating parasites with $10 \mathrm{nM}$ MitoTracker Red CMXROS (Invitrogen Life Technologies M7512) for 15 min prior to washout and imaging. 40-50 total parasites for each condition in two independent experiments were scored for focal or dispersed MitoTracker signal, and cell percentages were analyzed by two-tailed unpaired t-test in GraphPad Prism. For immunofluorescence assay (IFA) experiments, parasites were fixed, stained, and mounted, as previously described (Sigala et al., 2015; Tonkin et al., 2004). For IFA studies, the parasite mitochondrion was visualized using a polyclonal rabbit anti-HSP60 antibody (Novus NBP2-12734) and AlexaFluor 647-conjugated goat antirabbit $2^{\circ}$ antibody (Invitrogen Life Technologies A21244), the nucleus was stained with ProLong Gold Antifade Mountant with DAPI (Invitrogen Life Technologies P36931), and mACP-HA $\mathrm{A}_{2}$ was visualized with a Roche rat anti-HA monoclonal 3F10 primary antibody and FITC-conjugated donkey anti-rat $2^{\circ}$ antibody (Invitrogen Life Technologies A18746). Images were taken on DIC/brightfield, DAPI, GFP, and RFP channels using an EVOS M5000 imaging system or Zeiss 880 Laser-Scanning Confocal Microscope fitted with an Airyscan detector. Fiji/lmageJ was used to process and analyze images. All image adjustments, including contrast and brightness, were made on a linear scale.

\section{Rosetta modeling of the mACP-Isd11 interface}

A homology model of $P$. falciparum mACP bound to Isd11 was created using the InterPred modeling interface (Mirabello and Wallner, 2017) and the structure of bovine complex I (Zhu et al., 2016) that contained mACP bound to mammalian LYR proteins NDUFA6 and NDUFB9 (PDB 5LDW, chains T and W) as template. The homology models for ACP and Isd11 were then superimposed on the previously determined structure (Cory et al., 2017) of E. coli ACP bound to human Isd11 (PDB 5USR, chains L and D). The structural model for the parasite mACP-Isd11 complex was refined using Rosetta Dual Relax (Conway et al., 2014), which repeatedly alternates between coordinate minimization and sidechain packing 20 times with gradually increasing van der Waals repulsion strength. Four different modeling strategies were investigated that varied the template chains in 5 USR ( $L$ and $D$ or $J$ and $H$ ) and whether or not to restrain the refinement on the template coordinates. Ten independent refinements were performed for each modeling strategy and evaluated for Rosetta energy (Park et al., 2016). The unrestrained refinement of the starting model using chains $L$ and $D$ resulted in the final model of lowest calculated energy, and this model was used for further analysis. MacPyMOL (Schrodinger) version 1.8 was used for structural visualization.

\section{Recombinant protein expression in E. coli and purification}

Chemically competent $E$. coli BL21/DE3 cells were transformed alone or in combination with mACP$\mathrm{HA}_{2} /$ pET28a (full-length or the $\triangle 2-50$ truncation and WT or F113A mutant), ( $\left.\triangle 2-40\right)$ aACP-HA $/$ pET28a, Isd11-His $/$ /pET21d (WT, YR/AA, or LYR/AAA mutants), or full-length Nfs1-HA $2 /$ pET28a plasmids by heat shock. The bacteria were grown at $37^{\circ} \mathrm{C}$ in $20 \mathrm{~mL}$ LB media in the presence of ampicillin $(100 \mu \mathrm{g} / \mathrm{mL})$ or kanamycin $(50 \mu \mathrm{g} / \mathrm{mL})$. Bacterial cultures were allowed to grow to an optical density (at $600 \mathrm{~nm}$ ) of $0.4-0.6$ before inducing protein expression with isopropyl 1-thio- $\beta$-galactopyranoside (IPTG) (Goldbio 367931) at a final concentration of $1 \mathrm{mM}$. Induced cultures were grown overnight at $20^{\circ} \mathrm{C}$ before harvesting by centrifugation. Bacterial pellets were either used immediately or stored at $-20^{\circ} \mathrm{C}$. Bacterial pellets were resuspended in $1 \mathrm{~mL} 1 \times$ cold PBS and lysed by sonication on ice using a Branson sonicator equipped with a microtip for five sets of 10 pulses at $50 \%$ power and $50 \%$ duty cycle. Supernatants were clarified by centrifugation at 13,000 rpm for $10 \mathrm{~min}$. For purifying hexa-His-tagged proteins, $100 \mu \mathrm{L}$ of Ni-NTA resin (Thermo Scientific 88221) was equilibrated with $1 \mathrm{~mL}$ of buffer $\mathrm{A}\left(50 \mathrm{mM} \mathrm{NaH} \mathrm{PO}_{4} \cdot \mathrm{H}_{2} \mathrm{O}, 500 \mathrm{mM} \mathrm{NaCl}\right.$, and $5 \mathrm{mM}$ imidazole, $\mathrm{pH} 8.0$ ). $100 \mu \mathrm{L}$ of the clarified lysate supernatant was added to the equilibrated resin, diluted to $1 \mathrm{~mL}$ with buffer $A$, and incubated for $30 \mathrm{~min}$. Resin was collected by centrifugation and washed three times with buffer $A$ 
and once with wash buffer (Buffer A plus $50 \mathrm{mM}$ imidazole). Bound protein was eluted by incubating resin with $100 \mu \mathrm{L}$ elution buffer $B$ (Buffer $A$ plus $500 \mathrm{mM}$ imidazole) for $15 \mathrm{~min}$ on ice. To purify Isd11-

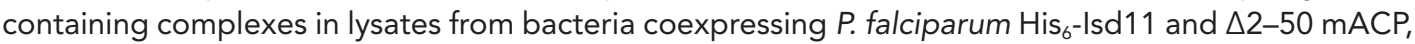
the imidazole eluate was run over an S-100 size-exclusion column (Cytiva Life Sciences 17116501) on an AKTA FPLC system (Cytiva Life Sciences). Lysates or eluates were diluted into SDS sample buffer and analyzed by SDS-PAGE and WB analysis.

\section{Anti-Nfs1 antibody validation}

The commercial rabbit anti-Nfs1 primary antibody (Abcam ab229829) was reported to be raised against the amino acids $208-457$ of human $\mathrm{Nfs} 1$, which is $55 \%$ identical to $P$. falciparum $\mathrm{Nfs} 1$ when aligned by BLAST. To determine if this commercial antibody selectively recognized $P$. falciparum Nfs 1 , we recombinantly expressed full-length $P$. falciparum Nfs1 or mACP in E. coli using the Nfs1-pET28a or mACPpET28a constructs with a $\mathrm{C}$-terminal dual $\mathrm{HA}_{2}$ tag (described above) that results in protein expression with expected sizes of $65 \mathrm{kDa}(\mathrm{Nfs} 1)$ or $21 \mathrm{kDa}$ (mACP). Protein expression was induced with IPTG, and bacteria were harvested by centrifugation and lysed in PBS by sonication. Bacterial lysate supernatants were fractionated by $10 \%$ SDS-PAGE by diluting $3 \mu \mathrm{L}$ of clarified lysate into $47 \mu \mathrm{L}$ of $1 \times$ SDS sample buffer, denaturing at $95^{\circ} \mathrm{C}$ for $10 \mathrm{~min}$, spinning at 13,000 rpm for $5 \mathrm{~min}$ to clarify, and loading samples onto a 10\% SDS-PAGE gel. After electrophoresis, samples were transferred to nitrocellulose membrane and probed with 1:1000 dilution of Roche Rat anti-HA primary antibody/1:10,000 dilution of goat anti-rat IRDye 680LT secondary antibody (LiCor 92668029) and 1:1000 dilution of rabbit anti-Nfs1 primary antibody /1:10,000 dilution of donkey anti-rabbit IRDye800CW secondary antibody (LiCor 92632213). Detection of a single band at $65 \mathrm{kDa}$ by both anti-HA and anti-Nfs1 antibodies confirmed the ability of these antibodies to recognize the HA-tagged $P$. falciparum Nfs1. The anti-Nfs 1 antibody did not recognize recombinant $m A C P-H A_{2}$, confirming its specificity, but did recognize an endogenous $E$. coli protein $\sim 48 \mathrm{kDa}$ that we identified by mass spectrometry as bacterial IscS, which is $60 \%$ identical to human Nfs1.

\section{Acknowledgements}

We thank Greg Ducker, Chris Hill, Roland Lill, Sara Nowinski, Jared Rutter, Dennis Winge, and members of the Sigala lab for helpful discussions and Sean Prigge for the plasmid encoding $P$. falciparum Isd11-GFP. We thank Rebecca Marvin for assistance with making the $P$. falciparum cytochrome $c_{1}$-GFP expression plasmid, Megan Okada for assistance with graphical figure schemes and the design of integration vectors, and Sandra Osburn for assistance with recombinant protein mass spectrometry. PAS holds a Career Award at the Scientific Interface from the Burroughs Wellcome Fund and a Pew Biomedical Scholarship from the Pew Charitable Trusts. SF was supported by the African American Doctoral Scholars Initiative at the University of Utah. Microscopy, flow cytometry, protein mass spectrometry, and DNA synthesis and sequencing were performed using core facilities at the University of Utah.

\section{Additional information}

Funding

\begin{tabular}{lll} 
Funder & Grant reference number & Author \\
\hline $\begin{array}{l}\text { National Institute of } \\
\text { General Medical Sciences }\end{array}$ & R35GM133764 & Paul A Sigala \\
\hline $\begin{array}{l}\text { National Institute of } \\
\text { General Medical Sciences }\end{array}$ & R01GM089778 & James A Wohlschlegel \\
\hline $\begin{array}{l}\text { National Institute of } \\
\begin{array}{l}\text { Diabetes and Digestive } \\
\text { and Kidney Diseases }\end{array}\end{array}$ & U54DK110858 & Paul A Sigala \\
\hline $\begin{array}{l}\text { Burroughs Wellcome Fund } \\
\text { 1011969 }\end{array}$ & \\
\hline \begin{tabular}{l} 
Pew Charitable Trusts \\
\hline
\end{tabular} & 32099 & Paul A Sigala \\
\hline
\end{tabular}




\begin{tabular}{lll} 
Funder & Grant reference number & Author \\
\hline $\begin{array}{l}\text { National Institute of } \\
\text { General Medical Sciences }\end{array}$ & T32GM122740 & Jaime Sepulveda \\
\hline $\begin{array}{l}\text { National Institutes of } \\
\text { Health }\end{array}$ & S100D018210 & Paul A Sigala \\
\hline
\end{tabular}

The funders had no role in study design, data collection and interpretation, or the decision to submit the work for publication.

Author contributions

Seyi Falekun, Conceptualization, Formal analysis, Investigation, Methodology, Validation, Visualization, Writing - original draft, Writing - review and editing; Jaime Sepulveda, Investigation, Methodology, Writing - review and editing; Yasaman Jami-Alahmadi, Hahnbeom Park, Formal analysis, Investigation, Writing - review and editing; James A Wohlschlegel, Funding acquisition, Project administration, Supervision; Paul A Sigala, Conceptualization, Formal analysis, Funding acquisition, Project administration, Supervision, Validation, Writing - original draft, Writing - review and editing

Author ORCIDs

Seyi Falekun (D) http://orcid.org/0000-0003-2280-4424

Jaime Sepulveda (iD http://orcid.org/0000-0002-3557-4093

Paul A Sigala (iD) http://orcid.org/0000-0002-3464-3042

Decision letter and Author response

Decision letter https://doi.org/10.7554/71636.sa1

Author response https://doi.org/10.7554/71636.sa2

\section{Additional files}

Supplementary files

- Supplementary file 1. PCR primers used for cloning and genotyping.

- Transparent reporting form

Data availability

All data reported or analyzed in this manuscript are available and included in the main and supplemental figures and in the source data files.

\section{References}

Adam AC, Bornhövd C, Prokisch H, Neupert W, Hell K. 2006. The Nfs1 interacting protein Isd11 has an essential role in Fe/S cluster biogenesis in mitochondria. The EMBO Journal 25: 174-183. DOI: https://doi.org/10.1038/ sj.emboj.7600905, PMID: 16341090

Allary M, Lu JZ, Zhu L, Prigge ST. 2007. Scavenging of the cofactor lipoate is essential for the survival of the malaria parasite Plasmodium falciparum. Molecular Microbiology 63: 1331-1344. DOI: https://doi.org/10.1111/ j.1365-2958.2007.05592.x, PMID: 17244193

Angerer H, Radermacher M, Mańkowska M, Steger M, Zwicker K, Heide H, Wittig I, Brandt U, Zickermann V. 2014. The LYR protein subunit NB4M/NDUFA6 of mitochondrial complex I anchors an acyl carrier protein and is essential for catalytic activity. PNAS 111: 5207-5212. DOI: https://doi.org/10.1073/pnas.1322438111, PMID: 24706851

Angerer H. 2015. Eukaryotic LYR Proteins Interact with Mitochondrial Protein Complexes. Biology 4: 133-150. DOI: https://doi.org/10.3390/biology4010133, PMID: 25686363

Antonova-Koch Y, Meister S, Abraham M, Luth MR, Ottilie S, Lukens AK, Sakata-Kato T, Vanaerschot M, Owen E, Jado JC, Maher SP, Calla J, Plouffe D, Zhong Y, Chen K, Chaumeau V, Conway AJ, McNamara CW, Ibanez M, Gagaring K, et al. 2018. Open-source discovery of chemical leads for next-generation chemoprotective antimalarials. Science 362: eaat9446. DOI: https://doi.org/10.1126/science.aat9446, PMID: 30523084

Atkinson A, Smith P, Fox JL, Cui TZ, Khalimonchuk O, Winge DR. 2011. The LYR protein Mzm1 functions in the insertion of the Rieske Fe/S protein in yeast mitochondria. Molecular and Cellular Biology 31: 3988-3996. DOI: https://doi.org/10.1128/MCB.05673-11, PMID: 21807901

Balu B, Shoue DA, Fraser MJ, Adams JH. 2005. High-efficiency transformation of Plasmodium falciparum by the lepidopteran transposable element piggyBac. PNAS 102: 16391-16396. DOI: https://doi.org/10.1073/pnas. 0504679102, PMID: 16260745 
Boniecki MT, Freibert SA, Mühlenhoff U, Lill R, Cygler M. 2017. Structure and functional dynamics of the mitochondrial Fe/S cluster synthesis complex. Nature Communications 8: 1287. DOI: https://doi.org/10.1038/ s41467-017-01497-1, PMID: 29097656

Braymer JJ, Freibert SA, Rakwalska-Bange M, Lill R. 2021. Mechanistic concepts of iron-sulfur protein biogenesis in Biology. Biochimica et Biophysica Acta. Molecular Cell Research 1868: 118863. DOI: https://doi.org/10. 1016/j.bbamcr.2020.118863, PMID: 33007329

Brown A, Rathore S, Kimanius D, Aibara S, Bai XC, Rorbach J, Amunts A, Ramakrishnan V. 2017. Structures of the human mitochondrial ribosome in native states of assembly. Nature Structural \& Molecular Biology 24: 866-869. DOI: https://doi.org/10.1038/nsmb.3464, PMID: 28892042

Cai K, Frederick RO, Tonelli M, Markley JL. 2017. Mitochondrial Cysteine Desulfurase and ISD11 Coexpressed in Escherichia coli Yield Complex Containing Acyl Carrier Protein. ACS Chemical Biology 12: 918-921. DOI: https://doi.org/10.1021/acschembio.6b01005, PMID: 28233492

Chan DI, Vogel HJ. 2010. Current understanding of fatty acid biosynthesis and the acyl carrier protein. The Biochemical Journal 430: 1-19. DOI: https://doi.org/10.1042/BJ20100462, PMID: 20662770

Conway P, Tyka MD, DiMaio F, Konerding DE, Baker D. 2014. Relaxation of backbone bond geometry improves protein energy landscape modeling. Protein Science 23: 47-55. DOI: https://doi.org/10.1002/pro.2389, PMID: 24265211

Cory SA, Van Vranken JG, Brignole EJ, Patra S, Winge DR, Drennan CL, Rutter J, Barondeau DP. 2017. Structure of human Fe-S assembly subcomplex reveals unexpected cysteine desulfurase architecture and acyl-ACP. ISD11 interactions. PNAS 114: E5325-E5334. DOI: https://doi.org/10.1073/pnas.1702849114, PMID: 28634302

Dellibovi-Ragheb TA, Gisselberg JE, Prigge ST. 2013. Parasites FeS up: iron-sulfur cluster biogenesis in eukaryotic pathogens. PLOS Pathogens 9: e1003227. DOI: https://doi.org/10.1371/journal.ppat.1003227, PMID: 23592980

Delves M, Plouffe D, Scheurer C, Meister S, Wittlin S, Winzeler EA, Sinden RE, Leroy D. 2012. The activities of current antimalarial drugs on the life cycle stages of Plasmodium: a comparative study with human and rodent parasites. PLOS Medicine 9: e1001169. DOI: https://doi.org/10.1371/journal.pmed.1001169, PMID: 22363211

Florens L, Carozza MJ, Swanson SK, Fournier M, Coleman MK, Workman JL, Washburn MP. 2006. Analyzing chromatin remodeling complexes using shotgun proteomics and normalized spectral abundance factors. Methods 40: 303-311. DOI: https://doi.org/10.1016/j.ymeth.2006.07.028, PMID: 17101441

Gallagher JR, Prigge ST. 2010. Plasmodium falciparum acyl carrier protein crystal structures in disulfide-linked and reduced states and their prevalence during blood stage growth. Proteins 78: 575-588. DOI: https://doi. org/10.1002/prot.22582, PMID: 19768685

Ganesan SM, Falla A, Goldfless SJ, Nasamu AS, Niles JC. 2016. Synthetic RNA-protein modules integrated with native translation mechanisms to control gene expression in malaria parasites. Nature Communications 7: 10727. DOI: https://doi.org/10.1038/ncomms10727, PMID: 26925876

Gisselberg JE, Dellibovi-Ragheb TA, Matthews KA, Bosch G, Prigge ST, Seeber F. 2013. The suf iron-sulfur cluster synthesis pathway is required for apicoplast maintenance in malaria parasites. PLOS Pathogens 9: e1003655. DOI: https://doi.org/10.1371/journal.ppat.1003655

Gomes AR, Bushell E, Schwach F, Girling G, Anar B, Quail MA, Herd C, Pfander C, Modrzynska K, Rayner JC, Billker O. 2015. A genome-scale vector resource enables high-throughput reverse genetic screening in a malaria parasite. Cell Host \& Microbe 17: 404-413. DOI: https://doi.org/10.1016/j.chom.2015.01.014, PMID 25732065

Goodman CD, Buchanan HD, McFadden GI. 2017. Is the Mitochondrion a Good Malaria Drug Target? Trends in Parasitology 33: 185-193. DOI: https://doi.org/10.1016/j.pt.2016.10.002, PMID: 27789127

Gully D, Moinier D, Loiseau L, Bouveret E. 2003. New partners of acyl carrier protein detected in Escherichia coli by tandem affinity purification. FEBS Letters 548: 90-96. DOI: https://doi.org/10.1016/s0014-5793(03)00746-4, PMID: 12885413

Haussig JM, Matuschewski K, Kooij TWA. 2014. Identification of vital and dispensable sulfur utilization factors in the Plasmodium apicoplast. PLOS ONE 9: e89718. DOI: https://doi.org/10.1371/journal.pone.0089718, PMID: 24586983

Herrera M.G, Pignataro MF, Noguera ME, Cruz KM, Santos J. 2018. Rescuing the Rescuer: On the Protein Complex between the Human Mitochondrial Acyl Carrier Protein and ISD11. ACS Chemical Biology 13: 1455-1462. DOI: https://doi.org/10.1021/acschembio.8b00184, PMID: 29737835

Herrera M, Noguera ME, Sewell KE, Agudelo Suárez WA, Capece L, Klinke S, Santos J. 2019. Structure of the Human ACP-ISD11 Heterodimer. Biochemistry 58: 4596-4609. DOI: https://doi.org/10.1021/acs.biochem. 9b00539, PMID: 31664822

Hiltunen JK, Schonauer MS, Autio KJ, Mittelmeier TM, Kastaniotis AJ, Dieckmann CL. 2009. Mitochondrial fatty acid synthesis type II: more than just fatty acids. The Journal of Biological Chemistry 284: 9011-9015. DOI: https://doi.org/10.1074/jbc.R800068200, PMID: 19028688

Janouškovec J, Tikhonenkov DV, Burki F, Howe AT, Kolísko M, Mylnikov AP, Keeling PJ. 2015. Factors mediating plastid dependency and the origins of parasitism in apicomplexans and their close relatives. PNAS 112: 10200-10207. DOI: https://doi.org/10.1073/pnas.1423790112, PMID: 25717057

Jayaraman V, Suryavanshi A, Kalale P, Kunala J, Balaram H. 2018. Biochemical characterization and essentiality of Plasmodium fumarate hydratase. The Journal of Biological Chemistry 293: 5878-5894. DOI: https://doi.org/10. 1074/jbc.M117.816298, PMID: 29449371 
Jhun H, Walters MS, Prigge ST. 2018. Using Lipoamidase as a Novel Probe To Interrogate the Importance of Lipoylation in Plasmodium falciparum. MBio 9: e01872. DOI: https://doi.org/10.1128/mBio.01872-18, PMID: 30459194

Ke H, Morrisey JM, Ganesan SM, Painter HJ, Mather MW, Vaidya AB. 2011. Variation among Plasmodium falciparum Strains in Their Reliance on Mitochondrial Electron Transport Chain Function. Eukaryotic Cell 10: 1053-1061. DOI: https://doi.org/10.1128/EC.05049-11, PMID: 21685321

Ke H, Sigala PA, Miura K, Morrisey JM, Mather MW, Crowley JR, Henderson JP, Goldberg DE, Long CA, Vaidya AB. 2014. The heme biosynthesis pathway is essential for Plasmodium falciparum development in mosquito stage but not in blood stages. The Journal of Biological Chemistry 289: 34827-34837. DOI: https:// doi.org/10.1074/jbc.M114.615831, PMID: 25352601

Ke H, Lewis IA, Morrisey JM, McLean KJ, Ganesan SM, Painter HJ, Mather MW, Jacobs-Lorena M, Llinás M, Vaidya AB. 2015. Genetic investigation of tricarboxylic acid metabolism during the Plasmodium falciparum life cycle. Cell Reports 11: 164-174. DOI: https://doi.org/10.1016/j.celrep.2015.03.011, PMID: 25843709

$\mathrm{Ke} \mathrm{H}$, Dass S, Morrisey JM, Mather MW, Vaidya AB. 2018. The mitochondrial ribosomal protein L13 is critical for the structural and functional integrity of the mitochondrion in Plasmodium falciparum. The Journal of Biological Chemistry 293: 8128-8137. DOI: https://doi.org/10.1074/jbc.RA118.002552, PMID: 29626096

Kim KS, Maio N, Singh A, Rouault TA. 2018. Cytosolic HSC20 integrates de novo iron-sulfur cluster biogenesis with the CIAO1-mediated transfer to recipients. Human Molecular Genetics 27: 837-852. DOI: https://doi.org/ 10.1093/hmg/ddy004, PMID: 29309586

Kispal G, Csere P, Prohl C, Lill R. 1999. The mitochondrial proteins Atm1p and Nfs $1 p$ are essential for biogenesis of cytosolic Fe/S proteins. The EMBO Journal 18: 3981-3989. DOI: https://doi.org/10.1093/emboj/18.14.3981, PMID: 10406803

Lill R, Freibert SA. 2020. Mechanisms of Mitochondrial Iron-Sulfur Protein Biogenesis. Annual Review of Biochemistry 89: 471-499. DOI: https://doi.org/10.1146/annurev-biochem-013118-111540, PMID: 31935115

MacRae JI, Dixon MWA, Dearnley MK, Chua HH, Chambers JM, Kenny S, Bottova I, Tilley L, McConville MJ. 2013. Mitochondrial metabolism of sexual and asexual blood stages of the malaria parasite Plasmodium falciparum. BMC Biology 11: 67-77. DOI: https://doi.org/10.1186/1741-7007-11-67, PMID: 23763941

Maio N, Rouault TA. 2020. Outlining the Complex Pathway of Mammalian Fe-S Cluster Biogenesis. Trends in Biochemical Sciences 45: 411-426. DOl: https://doi.org/10.1016/j.tibs.2020.02.001, PMID: 32311335

Majmudar JD, Feng X, Fox NG, Nabhan JF, Towle T, Ma T, Gooch R, Bulawa C, Yue WW, Martelli A. 2019. 4'-Phosphopantetheine and long acyl chain-dependent interactions are integral to human mitochondrial acyl carrier protein function. MedChemComm 10: 209-220. DOI: https://doi.org/10.1039/c8md00489g

Mamoun CB, Goldberg DE. 2001. Plasmodium protein phosphatase 2C dephosphorylates translation elongation factor 1 beta and inhibits its PKC-mediated nucleotide exchange activity in vitro. Molecular Microbiology 39: 973-981. DOI: https://doi.org/10.1046/j.1365-2958.2001.02289.x, PMID: 11251817

Mirabello C, Wallner B. 2017. terPred: A pipeline to identify and model protein-protein interactions. Proteins 85 : 1159-1170. DOl: https://doi.org/10.1002/prot.25280, PMID: 28263438

Nagaraj VA, Sundaram B, Varadarajan NM, Subramani PA, Kalappa DM, Ghosh SK, Padmanaban G. 2013. Malaria parasite-synthesized heme is essential in the mosquito and liver stages and complements host heme in the blood stages of infection. PLOS Pathogens 9: e1003522. DOI: https://doi.org/10.1371/journal.ppat. 1003522, PMID: 23935500

Nowinski SM, Van Vranken JG, Dove KK, Rutter J. 2018. Impact of Mitochondrial Fatty Acid Synthesis on Mitochondrial Biogenesis. Current Biology 28: R1212-R1219. DOI: https://doi.org/10.1016/j.cub.2018.08.022, PMID: 30352195

Nowinski SM, Solmonson A, Rusin SF, Maschek JA, Bensard CL, Fogarty S, Jeong MY, Lettlova S, Berg JA, Morgan JT, Ouyang Y, Naylor BC, Paulo JA, Funai K, Cox JE, Gygi SP, Winge DR, DeBerardinis RJ, Rutter J. 2020. Mitochondrial fatty acid synthesis coordinates oxidative metabolism in mammalian mitochondria. eLife 9: e58041. DOI: https://doi.org/10.7554/eLife.58041, PMID: 32804083

Oppenheim RD, Creek DJ, Macrae JI, Modrzynska KK, Pino P, Limenitakis J, Polonais V, Seeber F, Barrett MP, Billker O, McConville MJ, Soldati-Favre D. 2014. BCKDH: the missing link in apicomplexan mitochondrial metabolism is required for full virulence of Toxoplasma gondii and Plasmodium berghei. PLOS Pathogens 10: e1004263. DOI: https://doi.org/10.1371/journal.ppat.1004263, PMID: 25032958

Painter HJ, Morrisey JM, Mather MW, Vaidya AB. 2007. Specific role of mitochondrial electron transport in blood-stage Plasmodium falciparum. Nature 446: 88-91. DOI: https://doi.org/10.1038/nature05572, PMID: 17330044

Park H, Bradley P, Liu Y, Mulligan VK, Kim DE, Baker D, DiMaio F. 2016. Simultaneous Optimization of Biomolecular Energy Functions on Features from Small Molecules and Macromolecules. Journal of Chemical Theory and Computation 12: 6201-6212. DOI: https://doi.org/10.1021/acs.jctc.6b00819, PMID: 27766851

Park H, Ovchinnikov S, Kim DE, DiMaio F, Baker D. 2018. Protein homology model refinement by large-scale energy optimization. PNAS 115: 3054-3059. DOI: https://doi.org/10.1073/pnas.1719115115, PMID: 29507254

Qiu X, Janson CA. 2004. Structure of apo acyl carrier protein and a proposal to engineer protein crystallization through metal ions. Acta Crystallographica. Section D, Biological Crystallography 60: 1545-1554. DOI: https:// doi.org/10.1107/S0907444904015422, PMID: 15333924

Rathore A, Chu Q, Tan D, Martinez TF, Donaldson CJ, Diedrich JK, Yates JR, Saghatelian A. 2018. MIEF1 Microprotein Regulates Mitochondrial Translation. Biochemistry 57: 5564-5575. DOI: https://doi.org/10.1021/ acs.biochem.8b00726, PMID: 30215512 
Richards TA, van der Giezen M. 2006. Evolution of the Isd11-IscS complex reveals a single alpha-proteobacterial endosymbiosis for all eukaryotes. Molecular Biology and Evolution 23: 1341-1344. DOl: https://doi.org/10. 1093/molbev/msl001, PMID: 16648156

Rouault TA, Tong WH. 2005. Iron-sulphur cluster biogenesis and mitochondrial iron homeostasis. Nature Reviews. Molecular Cell Biology 6: 345-351. DOl: https://doi.org/10.1038/nrm1620, PMID: 15803140

Sharma AK, Pallesen LJ, Spang RJ, Walden WE. 2010. Cytosolic iron-sulfur cluster assembly (CIA) system: factors, mechanism, and relevance to cellular iron regulation. The Journal of Biological Chemistry 285: 26745-26751. DOI: https://doi.org/10.1074/jbc.R110.122218, PMID: 20522543

Shears MJ, Botté CY, McFadden GI. 2015. Fatty acid metabolism in the Plasmodium apicoplast: Drugs, doubts and knockouts. Molecular and Biochemical Parasitology 199: 34-50. DOI: https://doi.org/10.1016/j. molbiopara.2015.03.004, PMID: 25841762

Sidik SM, Huet D, Ganesan SM, Huynh MH, Wang T, Nasamu AS, Thiru P, Saeij JPJ, Carruthers VB, Niles JC, Lourido S. 2016. A Genome-wide CRISPR Screen in Toxoplasma Identifies Essential Apicomplexan Genes. Cell 166: 1423-1435.. DOI: https://doi.org/10.1016/j.cell.2016.08.019, PMID: 27594426

Sigala PA, Crowley JR, Henderson JP, Goldberg DE. 2015. Deconvoluting heme biosynthesis to target bloodstage malaria parasites. eLife 4: e09143. DOI: https://doi.org/10.7554/eLife.09143, PMID: 26173178

Skinner-Adams TS, Fisher GM, Riches AG, Hutt OE, Jarvis KE, Wilson T, von Itzstein M, Chopra P, Antonova-Koch Y, Meister S, Winzeler EA, Clarke M, Fidock DA, Burrows JN, Ryan JH, Andrews KT. 2019. Cyclization-blocked proguanil as a strategy to improve the antimalarial activity of atovaquone. Communications Biology 2: 166. DOI: https://doi.org/10.1038/s42003-019-0397-3, PMID: 31069275

Spillman NJ, Beck JR, Ganesan SM, Niles JC, Goldberg DE. 2017. The chaperonin TRiC forms an oligomeric complex in the malaria parasite cytosol. Cellular Microbiology 19: 12719. DOI: https://doi.org/10.1111/cmi. 12719, PMID: 28067475

Sturm A, Mollard V, Cozijnsen A, Goodman CD, McFadden GI. 2015. Mitochondrial ATP synthase is dispensable in blood-stage Plasmodium berghei rodent malaria but essential in the mosquito phase. PNAS 112: 1021610223. DOI: https://doi.org/10.1073/pnas.1423959112, PMID: 25831536

Tonkin CJ, van Dooren GG, Spurck TP, Struck NS, Good RT, Handman E, Cowman AF, McFadden GI. 2004. Localization of organellar proteins in Plasmodium falciparum using a novel set of transfection vectors and a new immunofluorescence fixation method. Molecular and Biochemical Parasitology 137: 13-21. DOI: https:// doi.org/10.1016/j.molbiopara.2004.05.009, PMID: 15279947

Vaca Jacome AS, Rabilloud T, Schaeffer-Reiss C, Rompais M, Ayoub D, Lane L, Bairoch A, Van Dorsselaer A, Carapito C. 2015. N-terminome analysis of the human mitochondrial proteome. Proteomics 15: 2519-2524. DOI: https://doi.org/10.1002/pmic.201400617, PMID: 25944712

van der Velden M, Rijpma SR, Russel FGM, Sauerwein RW, Koenderink JB. 2015. PfMDR2 and PfMDR5 are dispensable for Plasmodium falciparum asexual parasite multiplication but change in vitro susceptibility to anti-malarial drugs. Malaria Journal 14: 76. DOI: https://doi.org/10.1186/s12936-015-0581-y, PMID: 25884516

van Dooren GG, Stimmler LM, McFadden GI. 2006. Metabolic maps and functions of the Plasmodium mitochondrion. FEMS Microbiology Reviews 30: 596-630. DOI: https://doi.org/10.1111/j.1574-6976.2006. 00027.x, PMID: 16774588

van Schaijk BCL, Kumar TRS, Vos MW, Richman A, van Gemert G-J, Li T, Eappen AG, Williamson KC, Morahan BJ, Fishbaugher M, Kennedy M, Camargo N, Khan SM, Janse CJ, Sim KL, Hoffman SL, Kappe SHI, Sauerwein RW, Fidock DA, Vaughan AM. 2014. Type II fatty acid biosynthesis is essential for Plasmodium falciparum sporozoite development in the midgut of Anopheles mosquitoes. Eukaryotic Cell 13: 550-559. DOI: https://doi.org/10.1128/EC.00264-13, PMID: 24297444

Van Vranken JG, Jeong MY, Wei P, Chen YC, Gygi SP, Winge DR, Rutter J. 2016. The mitochondrial acyl carrier protein (ACP) coordinates mitochondrial fatty acid synthesis with iron sulfur cluster biogenesis. eLife 5: e17828. DOI: https://doi.org/10.7554/eLife.17828, PMID: 27540631

Van Vranken JG, Nowinski SM, Clowers KJ, Jeong MY, Ouyang Y, Berg JA, Gygi JP, Gygi SP, Winge DR, Rutter J. 2018. ACP Acylation Is an Acetyl-CoA-Dependent Modification Required for Electron Transport Chain Assembly. Molecular Cell 71: 567-580.. DOI: https://doi.org/10.1016/j.molcel.2018.06.039, PMID: 30118679

Vögtle F-N, Wortelkamp S, Zahedi RP, Becker D, Leidhold C, Gevaert K, Kellermann J, Voos W, Sickmann A, Pfanner N, Meisinger C. 2009. Global analysis of the mitochondrial N-proteome identifies a processing peptidase critical for protein stability. Cell 139: 428-439. DOI: https://doi.org/10.1016/j.cell.2009.07.045, PMID: 19837041

Wellems TE, Panton LJ, Gluzman IY, do Rosario VE, Gwadz RW, Walker-Jonah A, Krogstad DJ. 1990. Chloroquine resistance not linked to mdr-like genes in a Plasmodium falciparum cross. Nature 345: 253-255. DOI: https:// doi.org/10.1038/345253a0, PMID: 1970614

Wiedemann N, Urzica E, Guiard B, Müller H, Lohaus C, Meyer HE, Ryan MT, Meisinger C, Mühlenhoff U, Lill R, Pfanner N. 2006. Essential role of Isd11 in mitochondrial iron-sulfur cluster synthesis on Isu scaffold proteins. The EMBO Journal 25: 184-195. DOI: https://doi.org/10.1038/sj.emboj.7600906, PMID: 16341089

Wohlschlegel JA. 2009. Identification of SUMO-conjugated proteins and their SUMO attachment sites using proteomic mass spectrometry. Methods in Molecular Biology 497: 33-49. DOI: https://doi.org/10.1007/978-1. 59745-566-4_3, PMID: 19107409

Yu M, Kumar TRS, Nkrumah LJ, Coppi A, Retzlaff S, Li CD, Kelly BJ, Moura PA, Lakshmanan V, Freundlich JS, Valderramos J-C, Vilcheze C, Siedner M, Tsai JH-C, Falkard B, Sidhu ABS, Purcell LA, Gratraud P, Kremer L, Waters AP, et al. 2008. The fatty acid biosynthesis enzyme Fabl plays a key role in the development of 
liver-stage malarial parasites. Cell Host \& Microbe 4: 567-578. DOI: https://doi.org/10.1016/J.Chom.2008.11. 001, PMID: 19064257

Zara V, Palmisano I, Conte L, Trumpower BL. 2004. Further insights into the assembly of the yeast cytochrome bc1 complex based on analysis of single and double deletion mutants lacking supernumerary subunits and cytochrome b. European Journal of Biochemistry 271: 1209-1218. DOI: https://doi.org/10.1111/j.1432-1033. 2004.04024.x, PMID: 15009199

Zhang Z, Huang L, Shulmeister VM, Chi YI, Kim KK, Hung LW, Crofts AR, Berry EA, Kim SH. 1998. Electron transfer by domain movement in cytochrome bc1. Nature 392: 677-684. DOI: https://doi.org/10.1038/33612, PMID: 9565029

Zhang M, Wang C, Otto TD, Oberstaller J, Liao X, Adapa SR, Udenze K, Bronner IF, Casandra D, Mayho M, Brown J, Li S, Swanson J, Rayner JC, Jiang RHY, Adams JH. 2018. Uncovering the essential genes of the human malaria parasite Plasmodium falciparum by saturation mutagenesis. Science 360: eaap7847. DOI: https://doi. org/10.1126/science.aap7847

Zhu J, Vinothkumar KR, Hirst J. 2016. Structure of mammalian respiratory complex I. Nature 536: 354-358. DOI: https://doi.org/10.1038/nature19095, PMID: 27509854 